\title{
Pflanzensoziologische Untersuchungen der Waldgesellschaften des Riesengebirges
}

W. MATUSZKIEWICZ und A. MATUSZKIEWICZ

In Vorbereitung einer Vegetationskartierung des Nationalparks im Riesengebirge wurden im Jahre 1959 vegetationskundliche Untersuchungen angefangen, welche in zwei - drei Jahren abzuschliessen sind. Im vorliegenden Aufsatz wollen wir die Ergebnisse der pflanzensozioJogischen Durchforschung der Wald- und Gebüschgesellschaften aus den Klassen Vaccinio-Piceetea und Querco-Fagetea kurz darstellen.
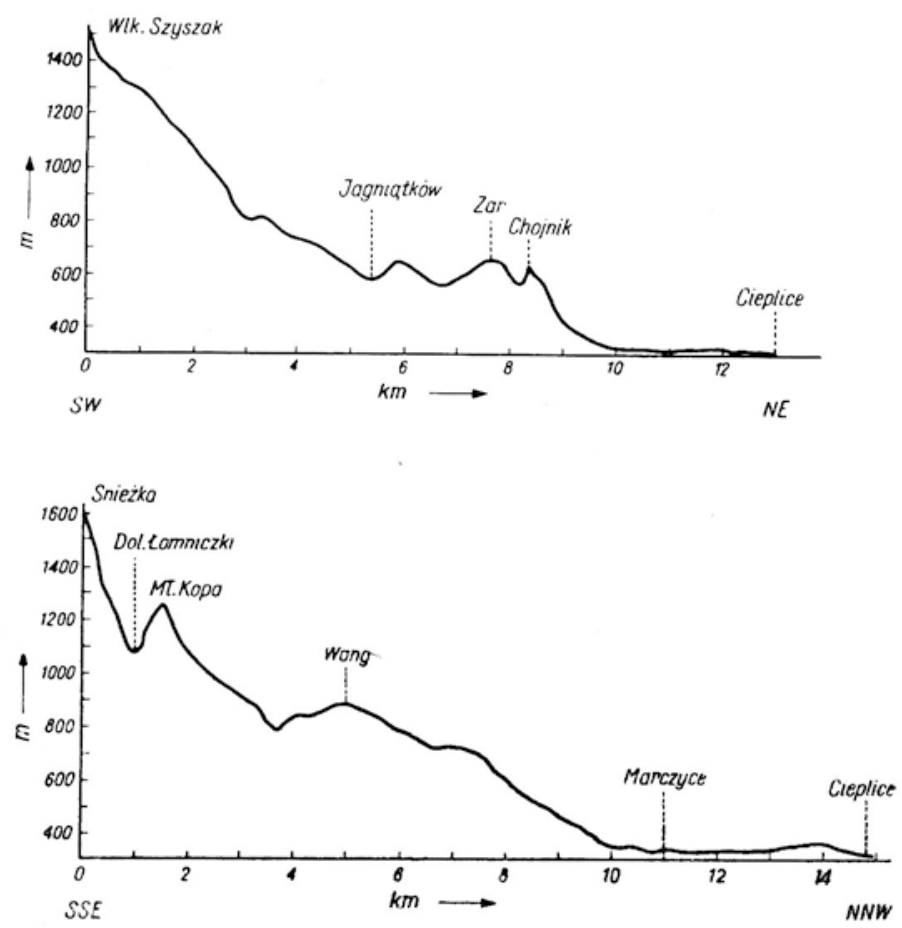

Fig. 1. Zwei topographische Querschnitte durch das nördliche Riesengebirge: Hohes Rad - Bad Warmbrunn (oben) und Schneekoppe - Bad Warmbrunn (unten) 


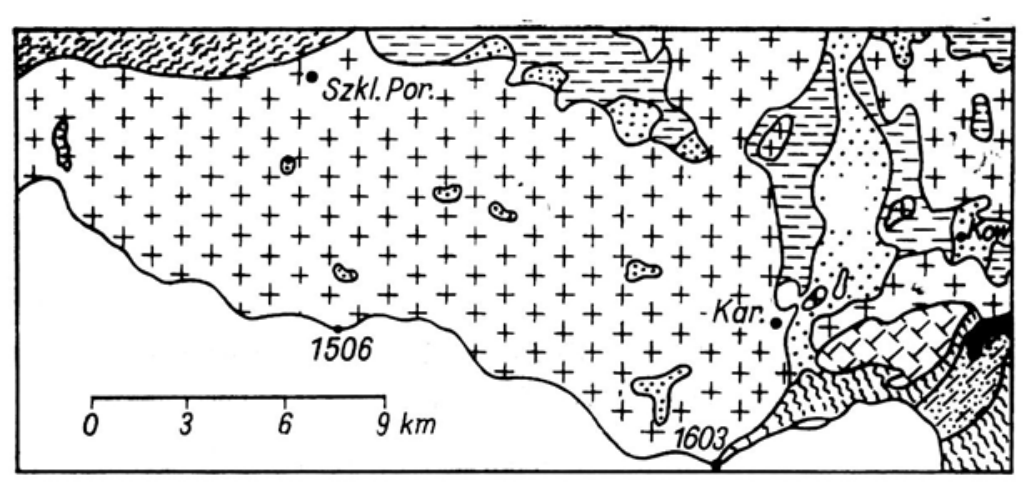

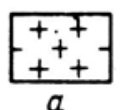

a
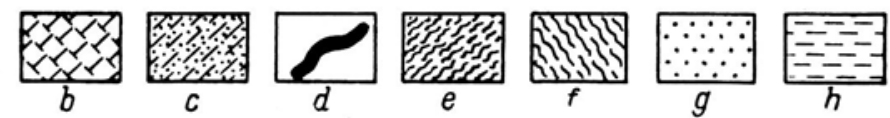

Fig. 2. Geologische Ubersichtskarte des schlesischen Teiles des Riesengebirges nach S. Doktorowicz-Hrebnicki (1948). a - Granit; b - Granitgneis, c - Biotitgneis; d - Amphibolit; e - Glimmerschiefer der Kontaktzone; $\mathrm{f}$ - Glimmerschiefer; g - Diluviale Sande und Kiese; h - Alluviale Maden und Fluszsande

Das Untersuchungsgebiet erstreckt sich über den schlesischen Teil des Hauptkammes vom Mummelberg zum Grenzbaudenpass und umfasst die ganze alpine und subalpine Stufe, den grössten Teil der oberen montanen Stufe sowie einzelne Gebiete im Bereich der unteren montanen Stufe, so besonders bei Szklarska Poręba (Schreiberhau), Jagniątków (Agnetendorf) und Karpacz (Krummhübel). Über die physiographischen Verhältnisse des Gebietes gibt es umfangreiche Literatur; einige standortsökologisch wichtigen Angaben sind in beiliegenden Diagrammen (Fig. 1-6) dargestellt worden.

In methodischer Hinsicht haben wir uns den Ideen der Schule von J. B r a u n-B l a n qu e t (1951) führen lassen. Bei Verwertung der Vegetationsaufnahmen und Fassung der Gesellschaftseinheiten haben wir das in der Bundesanstalt für Vegetationskartierung Stolzenau/Weser erarbeitete Verfahren mit Erfolg benutzt. Wir sind dem Leiter der BfV, Herrn Prof. Dr. Reinhold $\mathrm{T} u ̈ \mathrm{x}$ e n, zu einem ganz besonderen Dank verpflichtet für die freundliche Einladung und manche Anregungen sowie stete Fürsorge, welche wir während unseres Aufenthaltes in seinem Institut in Stolzen`u empfunden haben. Unser Dank gebührt auch den Mitarbeitern der BfV, den Herren Wilhelm Lohmeyer und Dr. Werner Trautmann, welche uns, trotz Belastung mit eigenen Arbeiten, durch ihre Erfahrung und manche Ratschläge vielfach unterstützt haben.

Neben der Vegetation wurden auch die Böden vergleichsmorphologisch untersucht und in die Beziehung mit den Pflanzengesellschaften gebracht. 
Bei der Deutung der bodenbildenden Prozesse sowie Fassung der Bodentypen folgen wir den Anschauungen von W. L. K u bi ë n a (1953) und W. L a at sch (1954). Für die schematischen Bodenprofile benutzen wir die Signaturen des Agrikulturchemischen Institutes ETH Zürich.

Die Pflanzengesellschaften des Riesengebirges sind schon früher von A. Zlatnik (1925, 1928), K. H u eck (1939), J. Wittig (1942/43), S. M a ck o (1952) u.a. untersucht worden ohne eine endgültige Lösung aller pflanzensoziologischen Fragen gefunden zu haben. Es ist das Ziel unserer Arbeit die Waldgesellschaften des betreffenden Gebietes auf Grund neugewonnenen Aufnahmematerials schärfer zu fassen, ihre Untereinheiten herauszuarbeiten und die Beziehungen zu den bestimmenden Standortsfaktoren aufzuhellen.

Wir haben 7 Assoziationen unterschieden, welche wir nun in systematischer Folge anführen wollen.

Klasse: VACCINIO-PICEETEA B r. - B 1. 1939.

Ordnung: PINETALIA O b e r d. 1949.

Verband: PINION L i b bert 1933.

Assoziation: PINETO-VACCINIETUM MYRTILLI (Kobendza 1930)

Br.-Bl. et Vlieg. 1939.

Der natürliche Kiefernwald kommt im Riesengebirge recht selten und nur in kleinen Fragmenten vor. Er besiedelt süd- und ostexponierte,

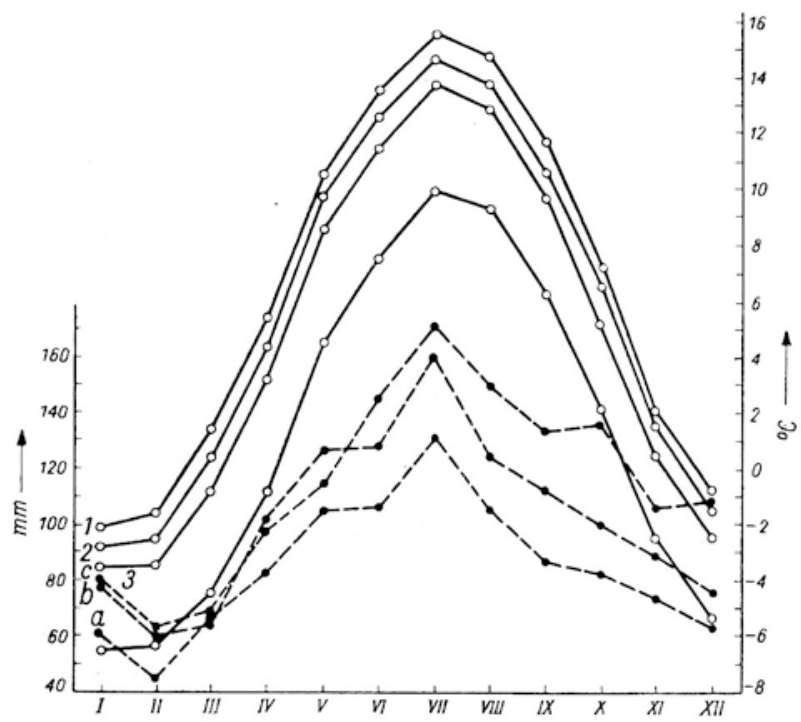

Fig. 3. Mittlere Monatstemperaturen: 1 - Krummhübel $(600 \mathrm{~m}) ; 2$ - Schreiberhau $(633 \mathrm{~m}) ; 3$ - Wang $(873 \mathrm{~m}) ; 4$ - Prinz-Heinrichbaude $(1415 \mathrm{~m})$. Mittlere monatliche Niederschlagssummen: a - Krummhübel $(600 \mathrm{~m})$; b - Wang $(873 \mathrm{~m})$; c - PrinzHeinrichbaude (1415 m) 


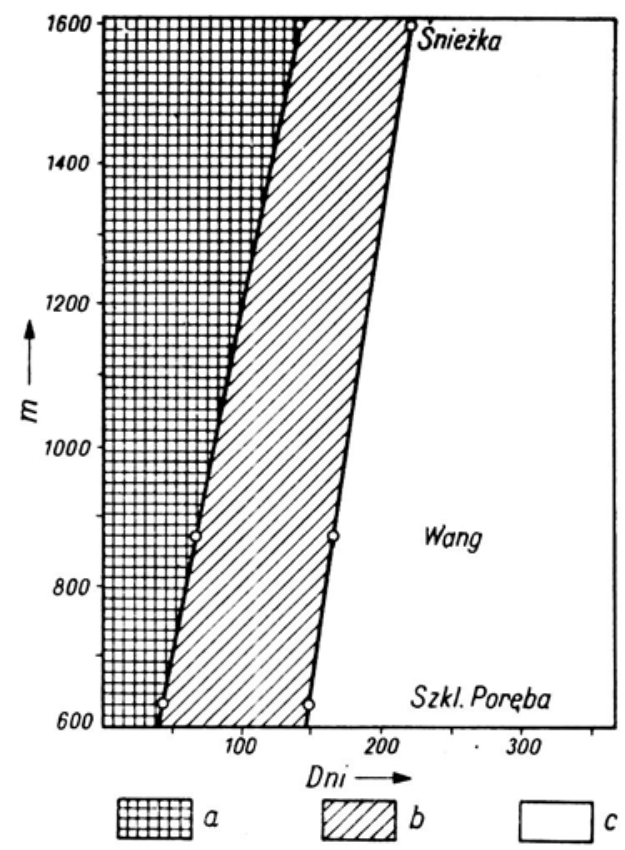

Fig. 4. Vertikale Verteilung der Eistage (a), Frostwechseltage (b) und frostfreien Tage (c) im Riesengebirge

Szklarska Porębo
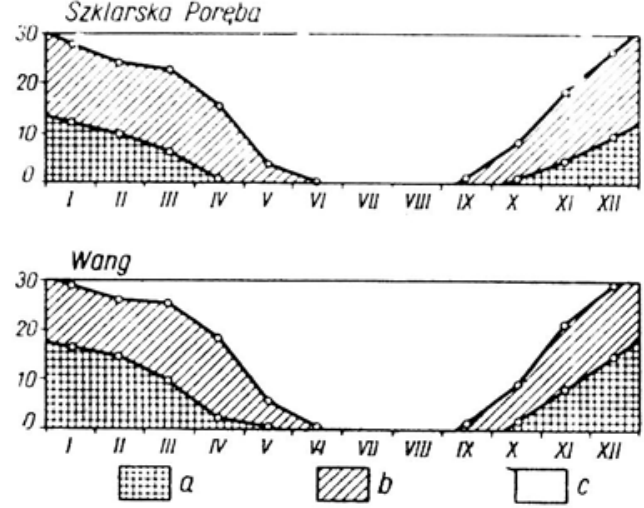

Fig. 5
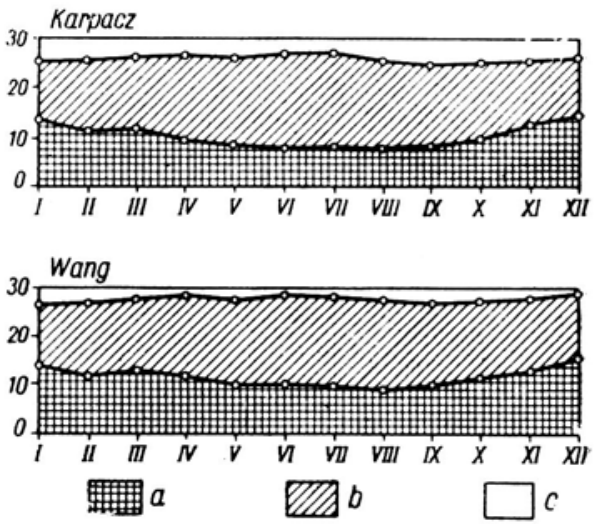

Fig. 6

Fig. 5. Jahreszeitliche Verteilung der Eistage (a), Frostwechseltage (b) und frostfreien Tage (c) in der montanen Stufe des Riesengebirges (oben: Schreiberhau, unten: Wang)

Fig. 6. Jahreszeitliche Verteilung des Bewölkungsgrades in der montanen Stufe des Riesengebirges (oben: Krummhübel, unten: Wang). a - völlig bewölkte Tage (mittlere Bewölkung 1,0-0,8); b - teilweise bewölkte Tage (mittlere Bewölkung $0,7-0,3) ; c-$ heitere Tage (mittlere Bewölkung $0,2-0,0$ ) 
felsblockreiche Kuppen in tieferen Lagen der unteren montanen Stufe. Es sind relativ die trockensten Waldstandorte des Gebietes.

Die Zugehörigkeit der Gesellschaft zum Pinion-Verband unterliegt keinem Zweifel, obwohl die entsprechenden Bestände, als extrazonale

Tabelle 1

Pineto-Vaccinietum myrtilli (Kobendza 19:0) B r.-Bl. et Vli e g. 1939

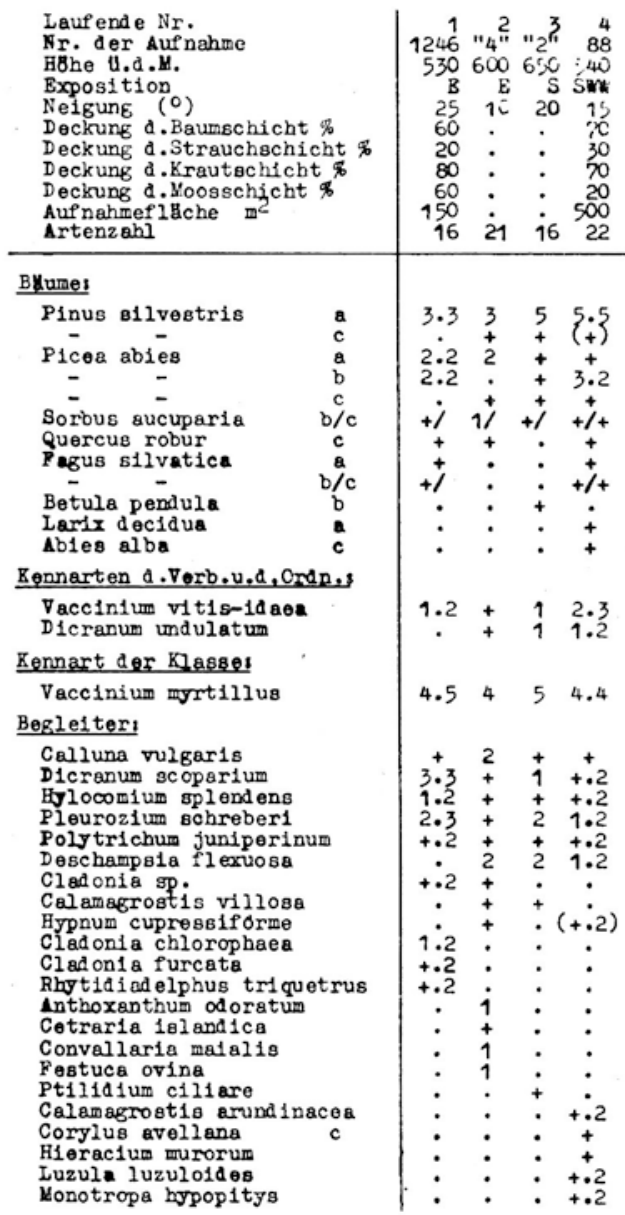

Randerscheinungen des Verbandes, stark verarmt sind und keine besseren Kennarten besitzen. Über die Assoziationszugehörigkeit lässt sich kaum etwas sicheres sagen solange nicht die vorgenommenen vergleichendsystematischen Untersuchungen die Frage nach der regionalen Gliedelung der mitteleuropäischen Kiefernwälder in Gebietsassoziationen gelöst haben. Wir haben vorläufig die Gesellschaft zum Pineto-Vaccinietum myrtilli im alten, weiten Sinne gestellt, sind jedoch überzeugt, dass 
Tabelle 2

Fichtenwälder und Fichtenforste der unteren montanen Stufe

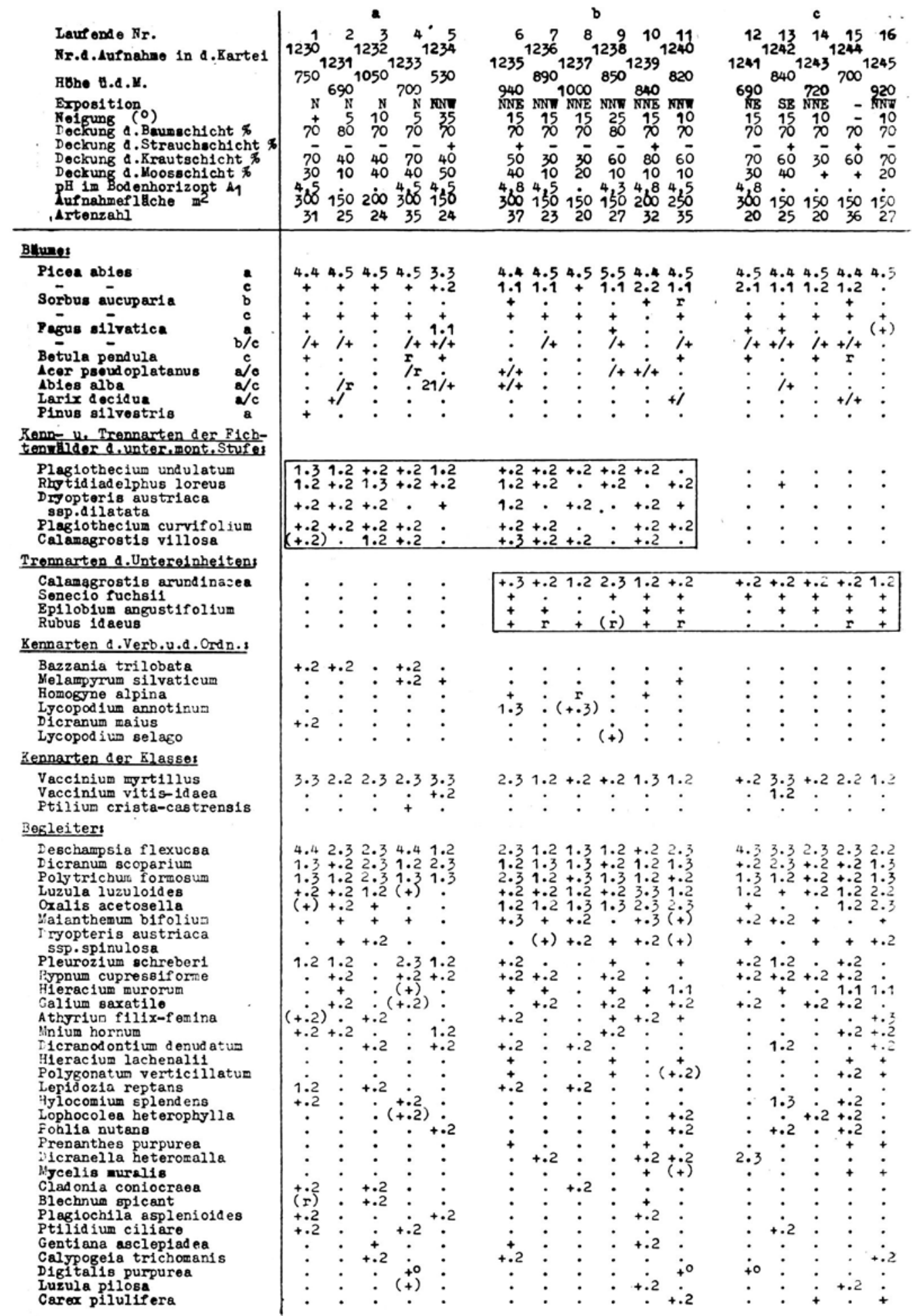


Tabelle 3

Gesellschaftsvergleich der natürlichen Fichtenwälder der oberen

(a) und unteren (b) montanen Stufe im Riesengebirge

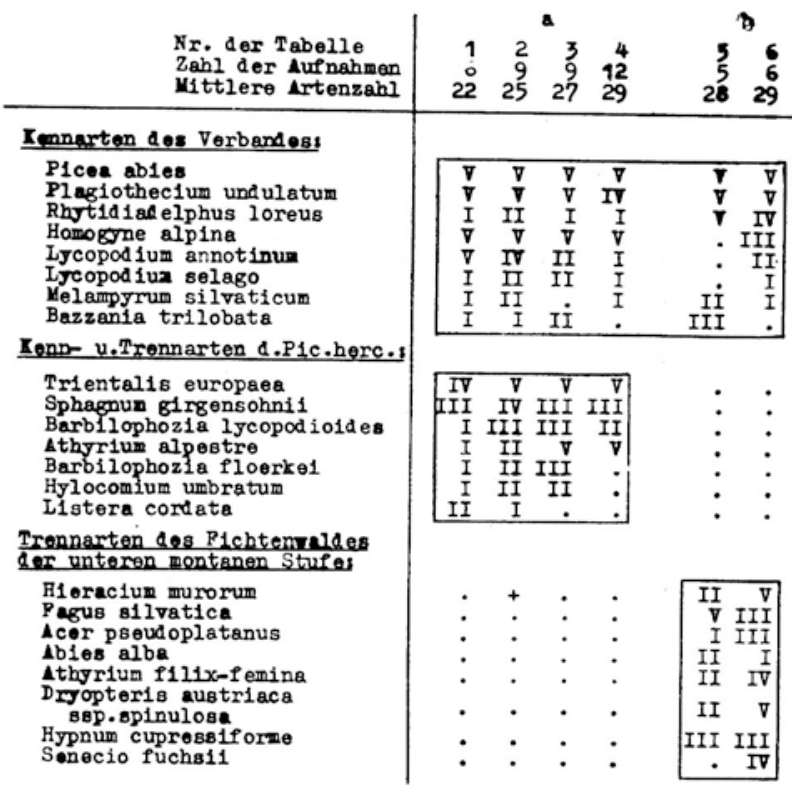

1 - Piceetum hercynicum typicum, reine Variante;

2 - Piceetum hercynicum typicum, Oxalis acetosella-Variante,

3 - Piceetum hercynicum filicetosum, reine Variante;

4 - Piceetum hercynicum filicetosum, Rumex arifolius-Variante;

5 - Fichtenwald der unteren montanen Stufe, reine Variante;

6 - Fichtenwald der unteren montanen Stufe, Calamagrostis arundinacea-Variante.

Fortsetzung der Tabelle 2:

$a$ - Fichtenwald, reine Variante

$b$ - Fichtenwald, Calamagrostis arundinacea-Variante

$c$ - Fichtenforst an Stelle des Hainsimsen-Buchenwaldes

Ausserdem in zwei Aufnahmen: Monotropa hypopitys: + (2, 14); Quercus robur c: $r$ (4), (5); Sambucus racemosa b: $+(6,10)$; Solidago virga-aurea: $+(6,10)$; Dryopteris filix-mas: $+\cdot 2(6),(+)(11)$; Cladonia digitata: $+\cdot 2(6,12)$; Polytrichum juniperinum: $+\cdot 2(7,15)$; Dryopteris phegopteris: $(+)(9),+\cdot 2(10)$; In einer Aufnahme: in 1 : Carex stellulata $+\cdot 2$, Mylia taylori $+\cdot 2$, Polytrichum commune $+\cdot 2$, Sphagnum rubellum $(+\cdot 2) ;$ in 2 : Ptilidium pulcherrimum $+\cdot 2$; in 3 : Luzula silvatica $+\cdot 2$; in 4 : Barbilophozia barbata $+\cdot 2$, B. hatche$\mathrm{ri}+\cdot 2$, Ctenidium molluscum $+\cdot 2$, Dicranum fuscescens $+\cdot 2$, Leucobryum glaucum $(\div \cdot \mathbf{2})$, Rhytidiadelphus triquetrus $+\cdot 2$, Salix caprea c $r$; in $5:$ Brachythecium starkei $+\cdot 2$, Polypodium vulgare + , Sphagnum nemoreum $+\cdot 2$; in $9:$ Mnium punctatum $+\cdot 2$; in 11 : Brachythecium rutabulum $+\cdot 2$; in 13 : Cladonia squamosa $+\cdot 2$, Isopterygium elegans $+\cdot 2$, Picea abies $b_{+}$; in 14: Cladonia sp. +2 ; in 15 : Carex pallescens $+\cdot 2$, Deschampsia caespitosa $+\cdot 2$, Lophocolea cuspidata $+\cdot 2$, Luzula multiflora $+\cdot 2$, Plagiothecium ruthei $+\cdot 2$; in 16: Dryopteris linnaeana + . 
diese Assoziation eher in zwei oder drei Gebietsassoziationen aufzuteilen und als Assoziationsgruppe aufzufassen ist. Dann würde unsere Gesellschaft der westlichsten jener Einheiten einzureihen.

\section{Ordnung: VACCINIO-PICEETALIA B r. - B 1. 1939.}

Verband: VACCINIO-PICEION B r. - B l. 1938.

Unterverband: ABIETO-PICEION B r.-B l. 1939.

Die ausgedehnten Fichtenwaldungen der unteren montanen Stufe des Riesengebirges wurden bisher durchwegs als künstliche Forste betrachtet. Das trifft in den meisten Fällen tatsächlich zu, doch gibt es auch Bestände, welche als lokalbedingte natürliche Dauergesellschaft aufzufassen sind (s. Tab. 2. a, b). Sie sind auf nordexponierte \pm flache Hänge oder tief eingeschnittene Bachtäler beschränkt und zeigen bei recht guter Vitalität keine Tendenz auf sich auszubreiten. Neben dominierender Fichte kommen im Baumbestande vereinzelte Buchen und Tannen vor. Als regionale Kennarten kommen für die Höhenstufe einige Verbands- und Ordnungsarten in Betracht, welche mit hoher Stetigkeit vorzukommen pflegen und die Gesellschaft gegenüber den Buchenwäldern sowie künstlichen Fichtenforsten gut abgrenzen lassen. Von dem Fichtenwald der oberen montanen Stufe wird die Gesellschaft durch Fehlen dessen Charakterarten sowie durch Vorhandensein übergreifender Querco-Fagetea-Arten und sonstiger Differentialarten (s. auch Tab. 3.) abgetrennt. Sie stellt bestimmt eine eigene Artenkombination dar und verdient den Rang einer Assoziation. Bei dem Fehlen entsprechender Vergleichsmaterialien aus anderen Teilen der Sudeten sowie benachbarten Gebirgen können wir nicht entscheiden ob hier eine eigene Gebietsassoziation vorliegt oder ob die Gesellschaft mit einer der schon bekannten Assoziationen zu identifizieren ist. Wir müssen uns vorläufig damit begnügen unsere Gesellschaft ihrer gesamten Artenkombination nach bloss dem Abieto-Piceion -. Unterverband anzuschliessen.

Die Abhängigkeit des Vorkommens der natürlichen Fichtenwälder von den topographischen Faktoren wird durch die Fig. 7. gezeigt.

Die Böden des Fichtenwaldes der unteren montanen Stufe sind als podsolierte Braunerden zu bezeichnen (s. Fig. 8.).

An Standortsformen der Gesellschaft sind zu unterscheiden:

a. ty pische Variante ohne eigene Trennarten und

b. Calamagrostis arundinacea - Variante, welche mit dem Waldreitgras sowie Senecio fuchsii, Prenanthes purpurea, Mycelis muralis, Dryopteris filix-mas u. a. zum Luzulo-Fagion vermittelt.

Zum Vergleich haben wir in Tab. 2. auch einige Aufnahmen echter Fichtenforsten zusammengestellt. 


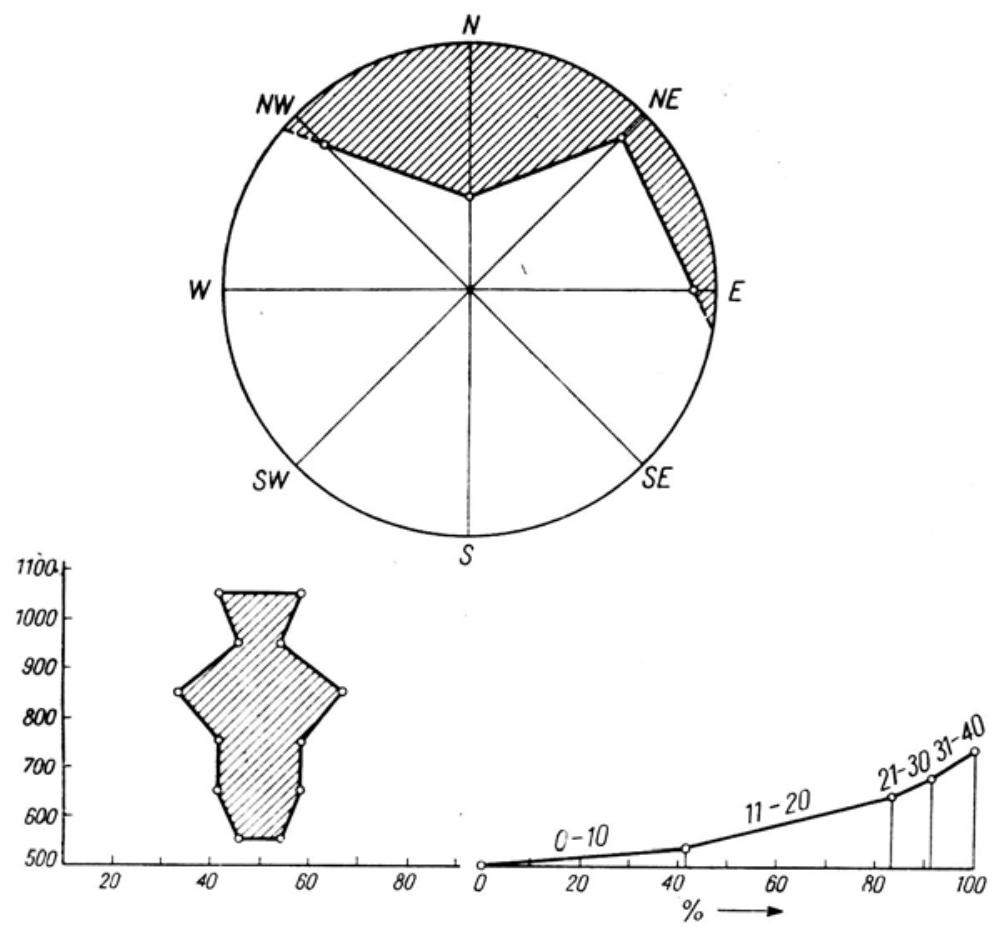

Fig. 7. Verteilung der Aufnahmen der natürlichen Fichtenwälder in der unteren montanen Stufe des Riesengebirges auf die verschiedenen Himmelsrichtungen, Bodenneigungen und Höhenlagen

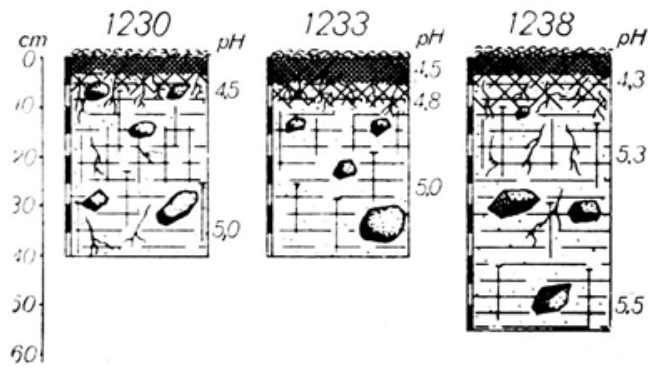

Fig. 8. Bodenprofile aus den natürlichen Fichtenwäldern der unteren montanen Stufe des Riesengebirges 
Unterverband: RHODORETO-VACCINION B r.-B l. 1926.

\section{Assoziation: PICEETUM HERCYNICUM Tüxen (1932) 1939.}

Natürliche und zum Teil urwüchsige Fichtenwälder bilden im Riesengebirge den zusammenhängenden Vegetationsgürtel in der oberen montanen Stufe. Sie stellen hier die klimabedingte Schlussgesellschaft dar. Durch die Charakterarten: Trientalis europaea, Sphagnum girgensohnii, Barbilophozia lycopodioides, B. floerkei, Hylocomium umbratum sowie einige subalpinen Begleiter (z. B. Athyrium alpestre) wird die Gesellschaft lokal gut umschrieben und gegen die Fichtenwälder der tieferen Lagen abgegrenzt; auch kommen die Laubwaldarten so gut wie gar nicht mehr vor. Die boreal-kontinentale Art Trientalis europaea kennzeichnet die Assoziation auch in regionaler Betrachtung.

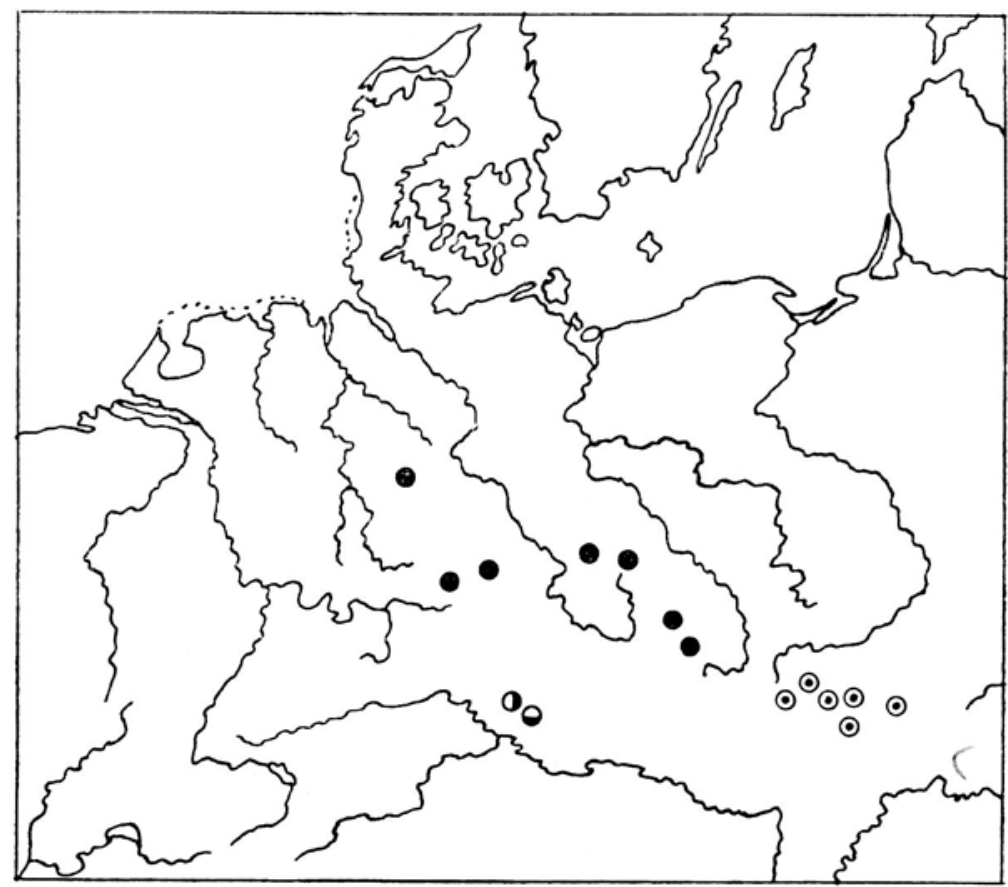

o $a$

$a b$

- $c$

$\odot d$

Fig. 9. Regionale Verteilung einiger Fichtenwälder der oberen montanen Stufe in IMitteleuropa: a - Mastigobryeto-Piceetum (Trautm. 1952) = SoldanelloPiceetum Volk 1939; b - Lophozieto-Piceetum Volk 1939; c - Piceetum hercynicum Tüx en (1932) 1937; d - Fichtenwälder der Westkarpathen („Piceetum tatricum" prov.) 


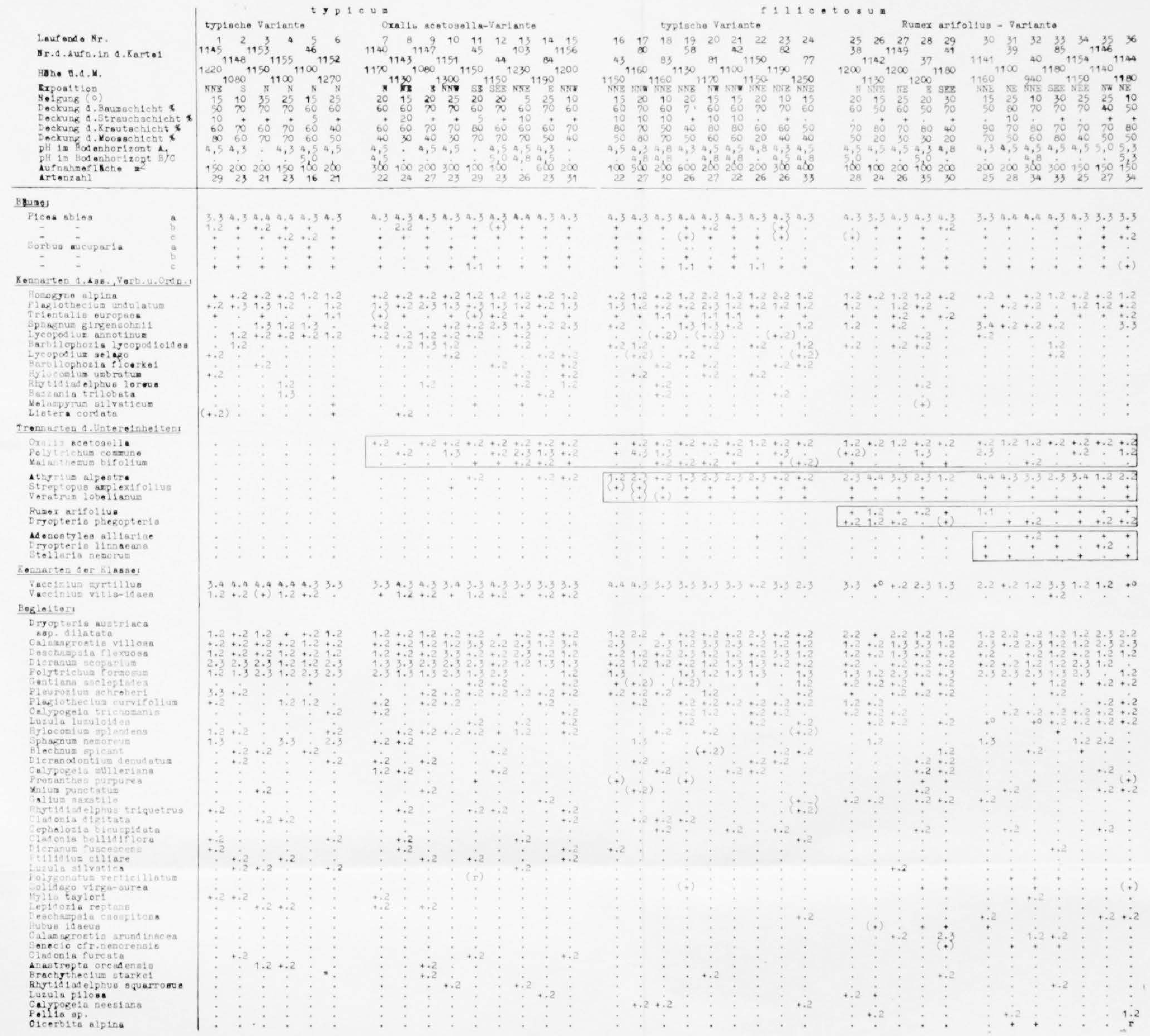

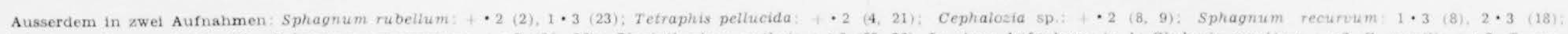

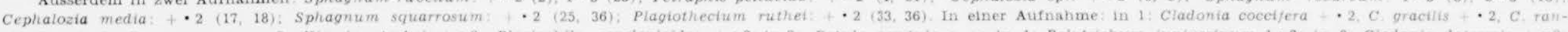

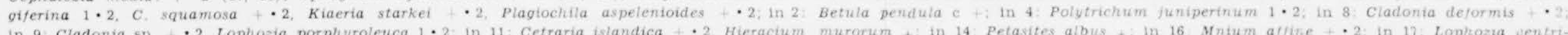

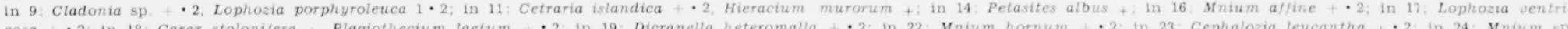

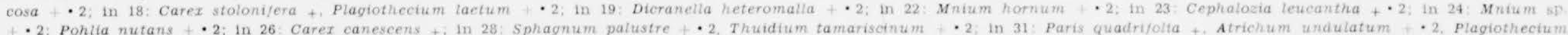

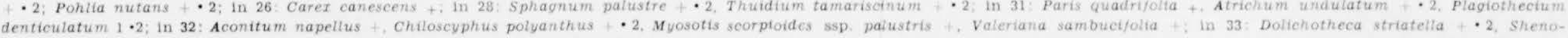
lobus minutus +2 , in 35 : Polytrtchum gractle $2 \cdot 2 ;$ in 36 : Paraleucobryim longifoltum $\cdot 2$, Plagiothecium platyphylium +2 . 
tentrionale“ (1. c. S. 61.) bezeichnet worden ist, doch dem mitteleuropäisch-alpinen Rhodoreto-Vaccinion-Unterverband und nicht dem borealnordischen Piceion septentrionale-Unterverband zu unterstellen, weil die floristisch-soziologischen, ökologischen und syngenetischen Beziehungen der Assoziation zu den subalpinen Klimaxwäldern viel enger sind, als schwache, bloss durch den Siebenstern etwa angedeutete Anknüpfungen an die zonalen Fichtenwälder der nordosteuropäischen Raumes. In dieser Hinsicht stimmt unsere Auffassung mit derjenigen von E. O berdorf e r (1957) überein.

Die systematische Stellung unserer Gesellschaft geht aus dem Vergleich mit den natürlichen Fichtenwäldern der Nachbargebiete klar hervor (s. Tab. 5, und Fig. 9.): sie ist derselben Assoziation anzuschliessen wie die subalpinen Fichtengesellschaften anderer Sudetenzüge, des Erzgebirges (Rheinhold F., 1939), des Thüringischen Schiefergebirges (G r ün ebe r g H., S chlüter H., 1957), des Harzes (Tüxen R., 1937) und wahrscheinlich auch des Oberpfälzer Waldes (O berdorfer E., 1957). Sie entsprechen alle dem Piceetum hercynicum $\mathrm{T} \ddot{\mathrm{x}} \mathrm{x}$ en (1932 n.n.) 1939. Die Gesellschaften des Bayerischen Waldes (V olk O. H., Priehäuser G., 1938; Trautmann W. 1952) sowie diejenigen der Westkarpathen (vgl. S z afer W., und Mitarbeitern 1923, 1927; P aw low ski B. u. Mitarb. 1927, 1928; W a la s J. 1933; M ed we ckaK orn ás A. 1955 u. a.) stellen dagegen andere Assoziationen dar.

Innerhalb des Piceetum hercynicum lassen sich wenigstens drei geographische Rassen vermuten:

a. Die Harz-Rasse: Homogyne alpina und Hylocomium umbratum fehlen, Listera cordata kommt kaum vor, die subatlantische Art Galium saxatile ist recht häufig; (Oberharz, Thüringer Wald).

b. Die Westsudeten-Rasse stellt die reinste Form der Assoziation dar; Vaccinio-Piceion - Arten sind am häufigsten vertreten (Iser- und Riesengebirge, Kolbenkamm).

c. Die Ostsudeten-Rasse vermittelt zu den Fichtenwäldern der Westkarpathen: Bazzania trilobata und Galium saxatile fehlen, Trientalis europaea wird seltener; Pyrola uniflora, welche ihren Schwerpunkt eher in karpathischen Piceeten hat, stellt sich ein (Glatzer Schneeberg, Altvatergebirge).

Piceetum hercynicum bildet im Riesengebirge eine Vegetationsstufe zwischen $\pm 1000-1250 \mathrm{~m}$ ü. d. M. Die tiefsten Bestände kennen wir etwa um $940 \mathrm{~m}$; es handelt sich um nordexponierte, luftkalte Täler. Die obere Grenze der Gesellschaft, welche mit der subalpinen Waldgrenze zusammenfällt, liegt etwa um $1250-1260 \mathrm{~m}$; an besonders günstigen Stellen sind recht gut entwickelte Bestände noch bei $1300 \mathrm{~m}$ ü. d. M. 


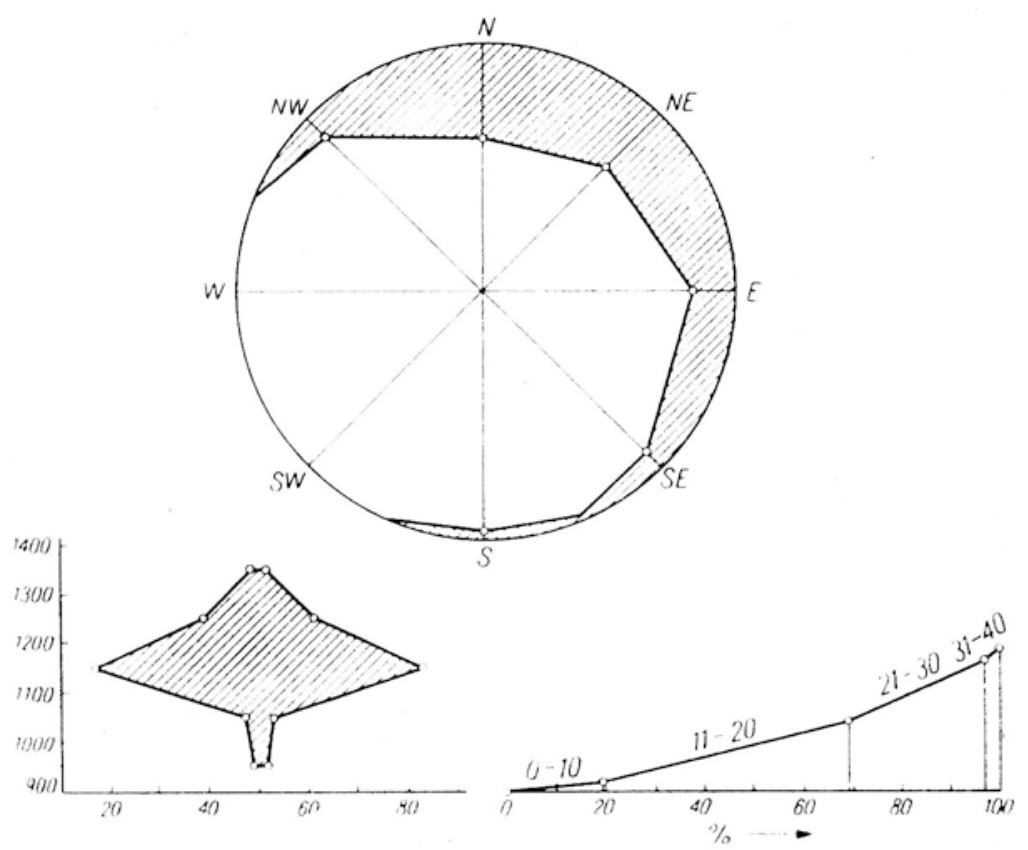

Fig. 10. Verteilung der Aufnahmen des Piceetum hercynicum im Riesengebirge auf die verschiedenen Himmelsrichtungen, Bodenneigungen und Höhenlagen

anzutreffen. Im optimalen Verbreitungsbereich ist die Gesellschaft wenig von topographischen Standortsverhältnissen abhängig, wohl dagegen in extremen Lagen. Auch stellt sich die Gesellschaft, als gesamte Assoziation betrachtet, auf verschiedenen Böden ein (Fig. 11.). Es sind am häufigsten primäre Eisen-Humus-Podsole vom verschiedenen Entwicklungsgrad, doch gibt es auch oligotrophe Ranker, gleyartige- und Gley-Podsole, Stagnogley-Böden und selbst Torfböden. Die Assoziation ist also als klimatisch bedingte Schlussgesellschaft aufzufassen.

Es lassen sich folgende Untereinheiten unterscheiden:

A. Piceetum hercynicum typicum - typische Subassoziation ohne eigene Trennarten. In der Krautschicht dominieren meist die Vaccinien; auch azidophile Gräser Deschampsia flexuosa und Calamagrostis villosa. können faziesbildend auftreten. Die Kennarten des Verbandes und der Ordnung sind reichlich vorhanden. Diese Form ist für die oberen Hanglagen sowie skelletreiche, konvexe Hänge mit guter Wasserabführung charakteristisch. Man kann unterscheiden:

a. reine Variante auf relativ trockenen, feinerdearmen Felsblockböden und 
b. Oxalis-Variante mit Frischezeigern Oxalis acetosella, Maianthemum bifolium und Polytrichum commune als Trennarten. Die letztgenannte Form kommt auf etwas frischeren, mittelkörnigen Böden vor und leitet zu der nächsten Untergesellschaft über.

B. Piceetum hercynicum filicetosum - die farnreiche Subassoziation - wird in ihrem Aspekt durch die meist faziesbildenden Farne Athyrium alpestre und Dryopteris austriaca ssp. dilatata bestimmt. Als Trennarten sind die aus Hochstaudenfluren der Betulo-Adenostyletea Klasse übergreifenden Arten zu nennen. Die Gesellschaft stockt auî feuchten, mit fliessendem Wasser durchrieselten Böden und ist als lokalbedingte Dauergesellschaft aufzufassen. Sie kommt in flachen Mulden, weiten Bachrinen und anderen wasserzügigen, „konkaven“ Hangteilen vor, Für das Gedeihen dieser Einheit ist entsprechende Boden- und Luftfeuchtigkeit von ausschlaggebender Bedeutug. Die farnreiche Subassoziation des subalpinen Fichtenwaldes ist daher in höheren Lagen relativ häufiger, doch kann die Gesellschaft längst der Bachrinnen ziemlich tief hinabsteigen.

Wir haben zwei Ausbildungsformen unterschieden:

a. Reine Variante steht dem Typus der Gesellschaft noch recht nahe. Der Kronenschluss ist normal, in der Krautschicht herrscht noch die Heidelbeere und andere Vaccinio-Piceetea-Arten vor. Der Alpen-
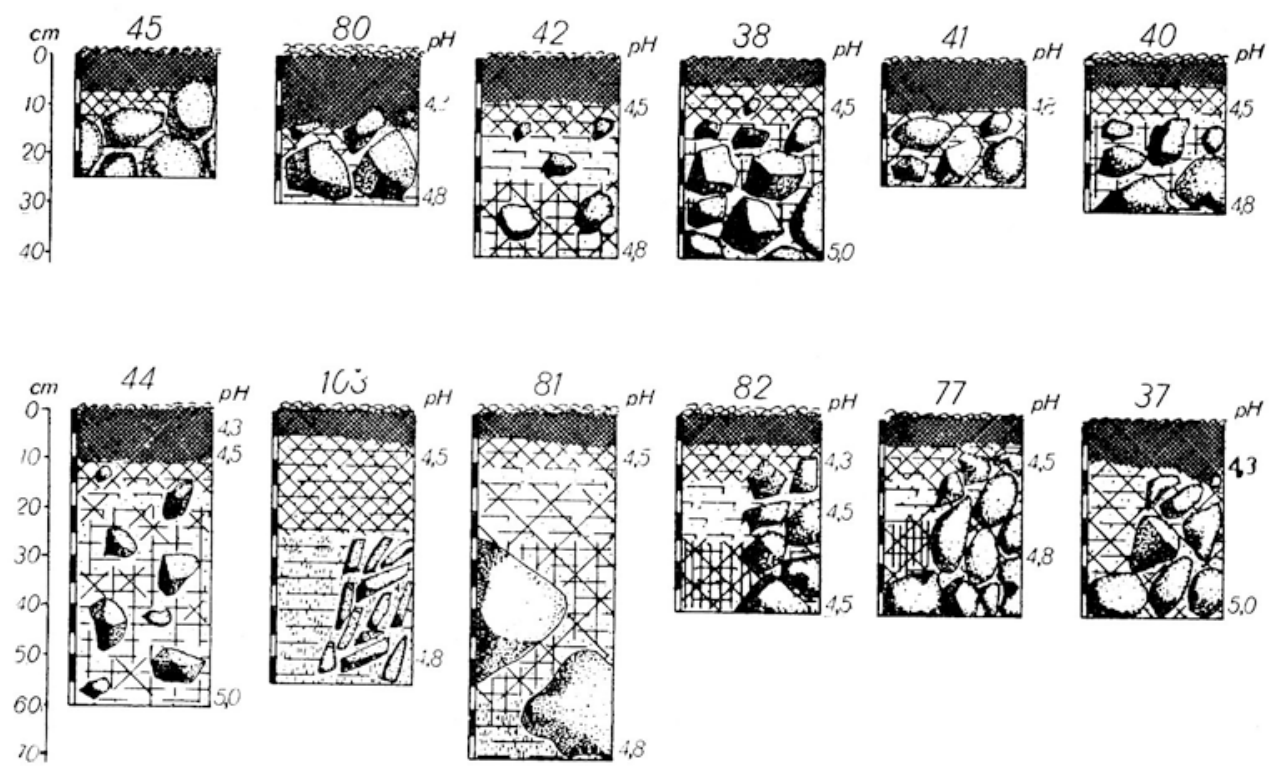

Fig. 11. Bodenprofile aus dem Piceetum hercynicum des Riesengebirges 
Tabelle 6

Piceetum hercynicum sphagnetosum Tüxen 1937

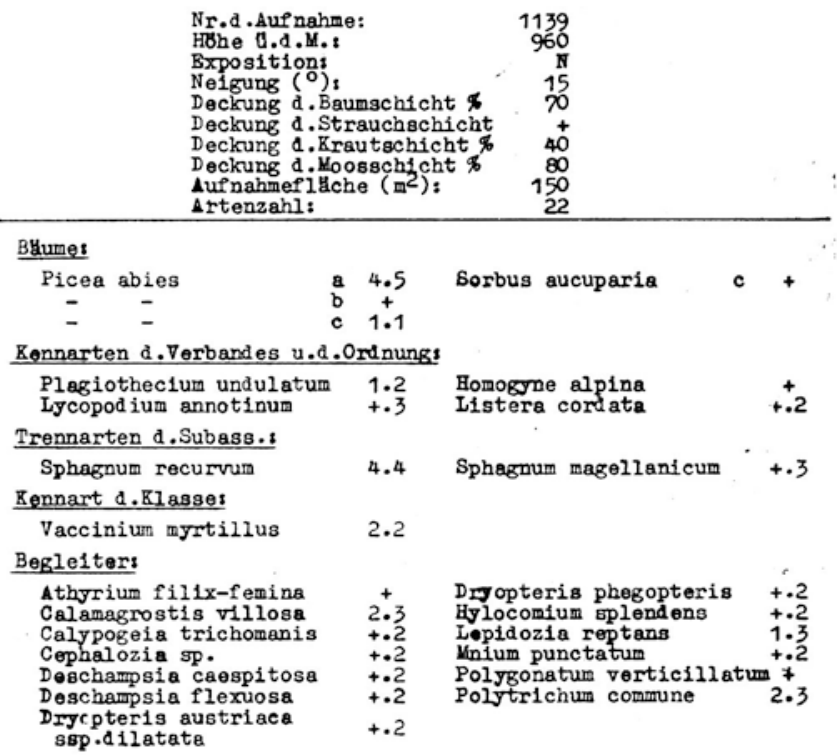

frauenfarn ist stets vorhanden, spielt jedoch mengenmässig eine untergeordnete Rolle. Die Gesellschaft ist für feinerdereiche, mässig feuchte Standorte typisch.

b. Die Variante von Rumex arifolius und Dryopteris phegopteris stellt auf wasserüberrieselten Böden eine Übergangsform zwischen dem Fichtenwald und Hochstaudenfluren, welche als Kontaktgesellschaften oft im kleinflächigen Wechsel vorzukommen pflegen, dar. Der Kronenschluss löst sich etwas auf; die Charakterzüge des Vaccinio-Piceion-Verbandes werden abgeschwächt. Die Gesellschaft ist bei zusagenden Reliefverhältnissen besonders gegen die Waldgrenze zu Hause und bestimmt oft das Landschaftsbild in höchsten Lagen der oberen montanen Stufe.

C. Piceetum hercynicum sphagnetosum - die moosreiche Subassoziation -- welche z. B. im Oberharz zu den bezeichnendsten Ausbildungen der Assoziation gehört, kommt im Riesengebirge nur kleinflächig in abflusslosen, versumpfenden Mulden vor. Sie wird durch die aspektbestimmenden Sphagnum-Polstern und Polytrichum commune-Teppiche gekennzeichnet und leitet mit einigen Ledetalia-Arten $\mathrm{zu}$ den hochmoorartigen, auf ihre systematische Stellung noch zu untersuchenden, Gesellschaften über. 


\section{Assoziation: PINETUM MUGHI SUDETICUM ass. nova}

Ausgedehnte Legföhrenbestände, welche im Riesengebirge in einem Gürtel oberhalb der Waldgrenze vorkommen, wurden bisher mehrfach beschrieben, ohne dass man auf ihre pflanzensoziologisch-systematische Fassung näher eingegangen wäre. Die Formation des Knieholzes ist fremd den anderen Mittelgebirgen Europas und auch dem grössten Teil der Alpen. Aus den Karpathen, wo die Gesellschaft grossflächig und vielerorts landschaftsbeherrschend vorkommt ist die Assoziation Pinetum mughi carpaticum Paw low ski 1927 beschrieben worden. Es bleibt zu entscheiden, ob wir im Riesengebirge mit derselben oder einer vikarierenden Gebietsassoziotion zu tun haben. Auf Grund des Vergleiches beider Gesellschaften (s. Tab. 8.) kommen wir zu folgenden Schlüssen:

1. Die Ausbildung vom Riesengebirge ist floristisch bedeutend ärmer als jene der Karpathen.
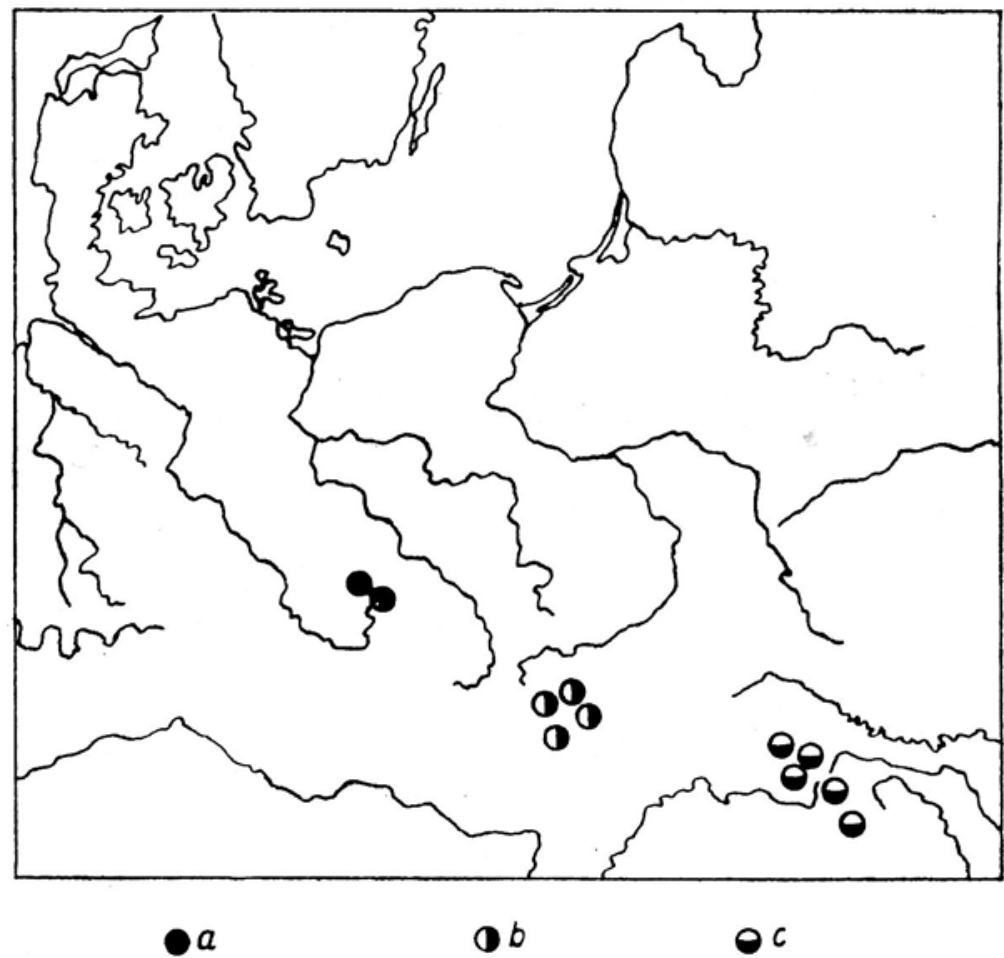

ob

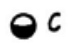

Fig. 12. Regionale Verteilung einiger subalpinen Knieholzgesellschaften im östlichen Teil Mitteleuropas. a - Pinetum mughi sudeticum; b - Pinetum mughi carpaticum, Rasse der Westkarpathen; c - Pinetum mughi carpaticum, Rasse der Ostkarpathen 


\begin{tabular}{|c|c|c|c|}
\hline 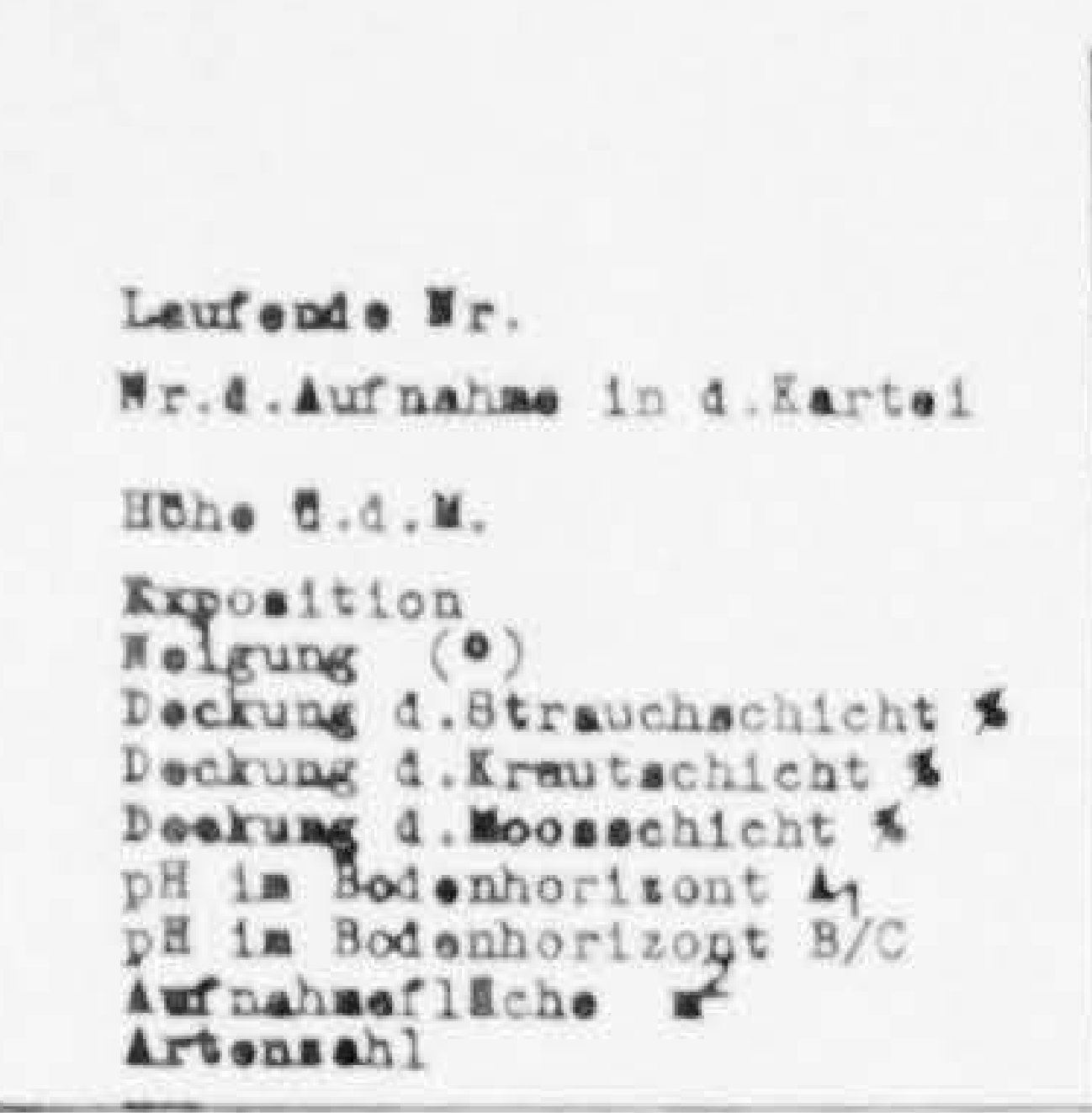 & 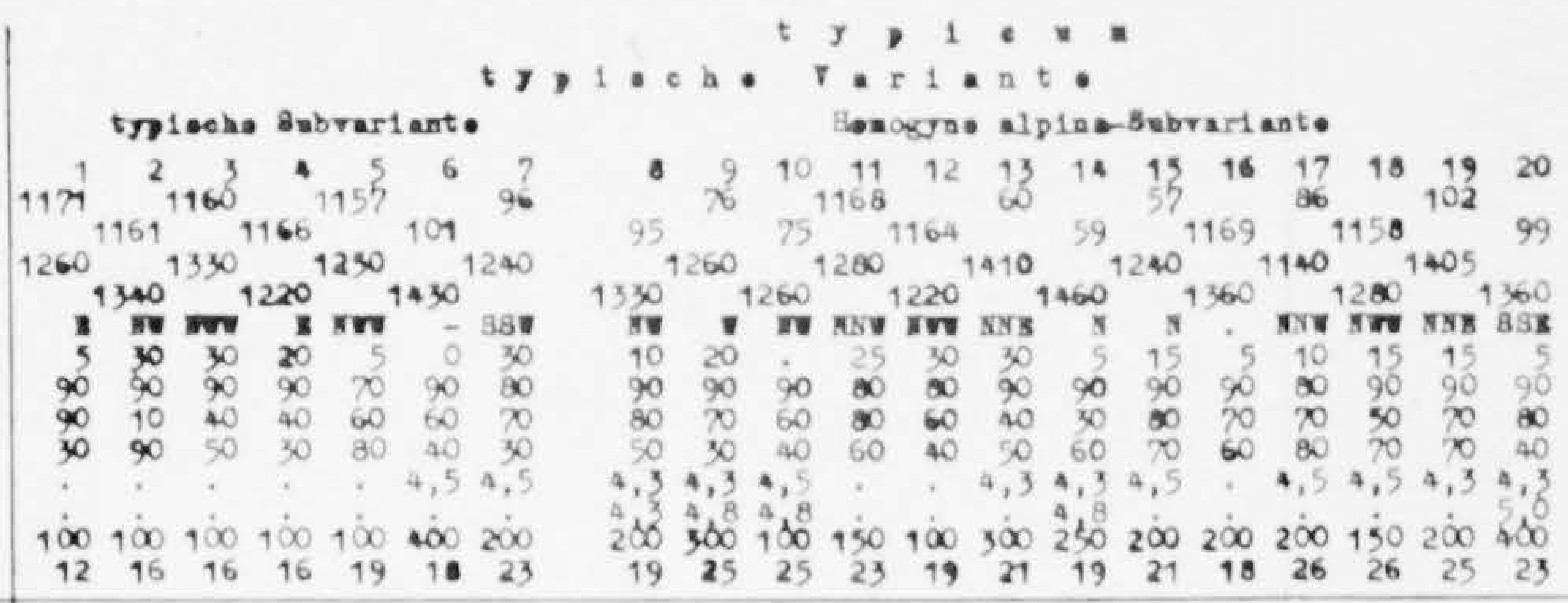 & 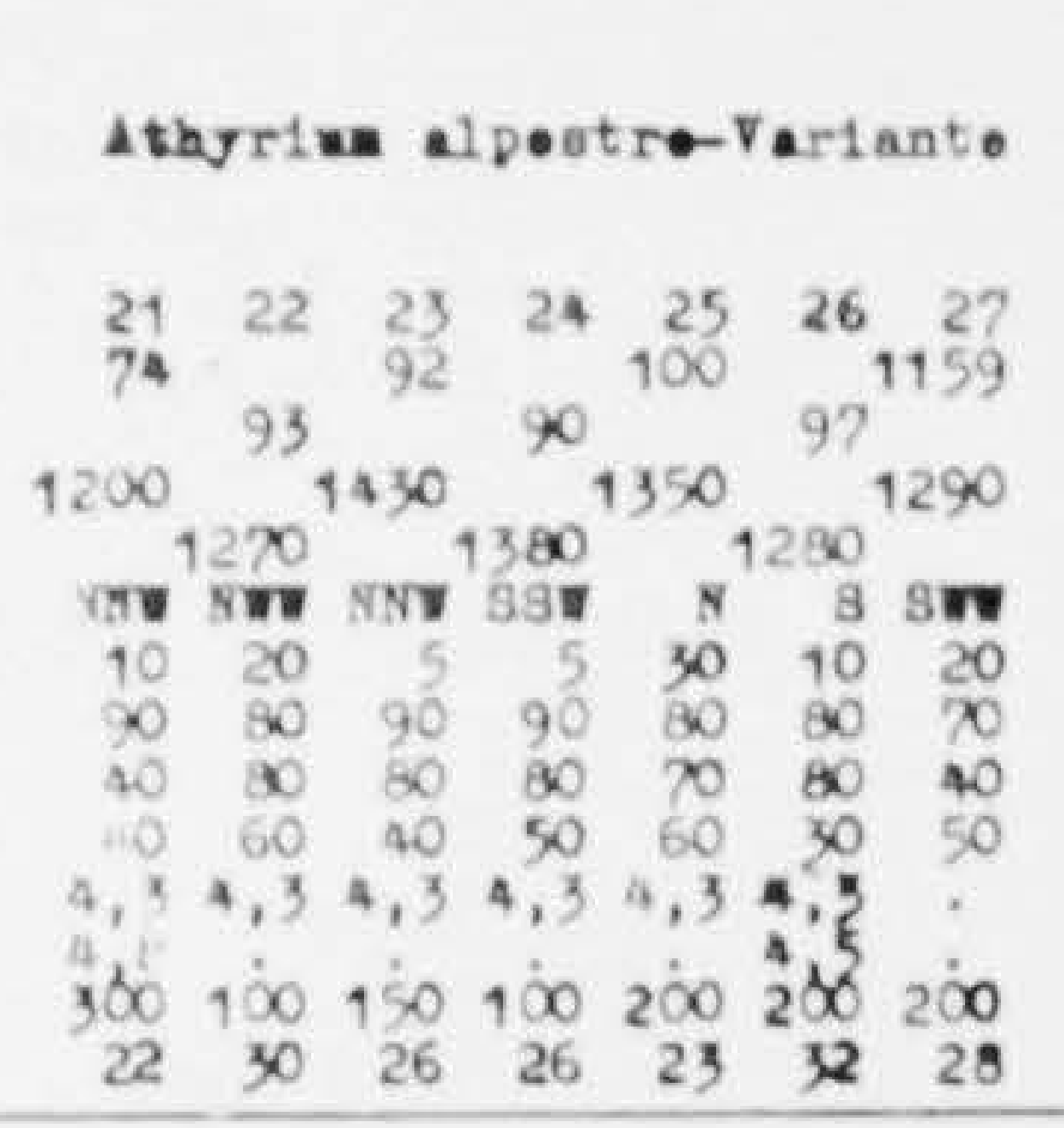 & $\begin{array}{r}r 9 \\
15 \\
80 \\
90 \\
60 \\
4,3 \\
300 \\
35 \\
35\end{array}$ \\
\hline 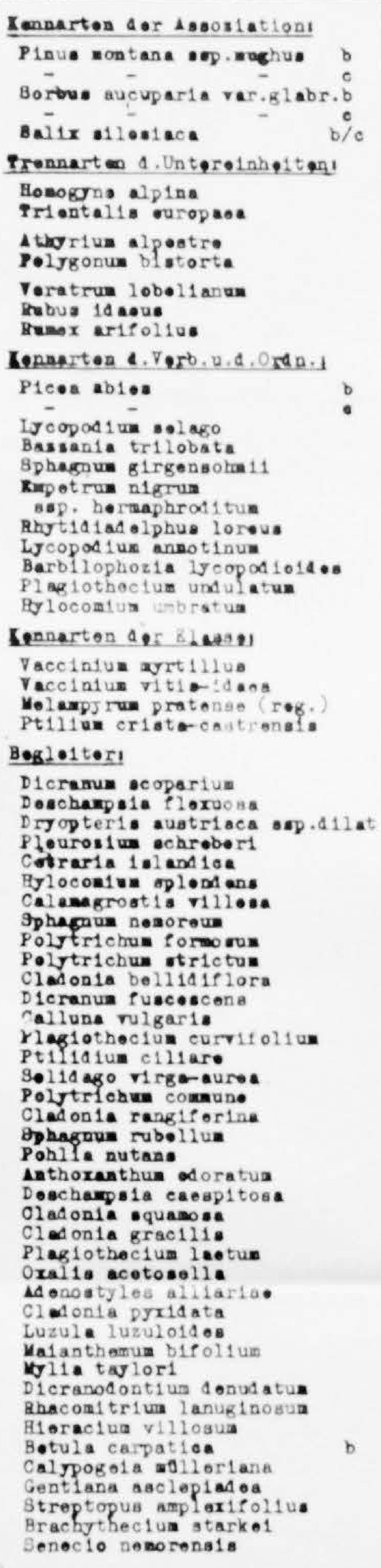 & 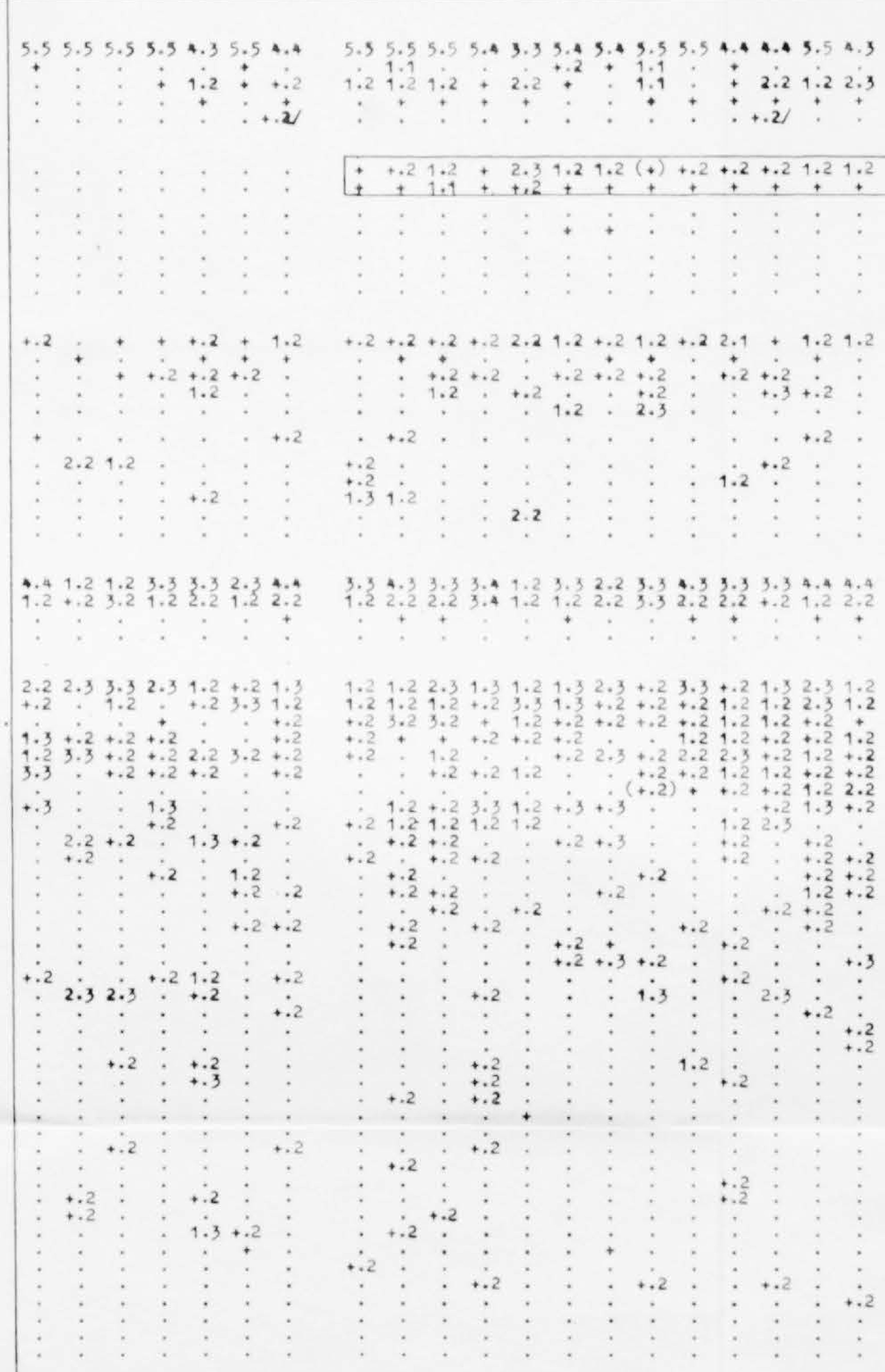 & 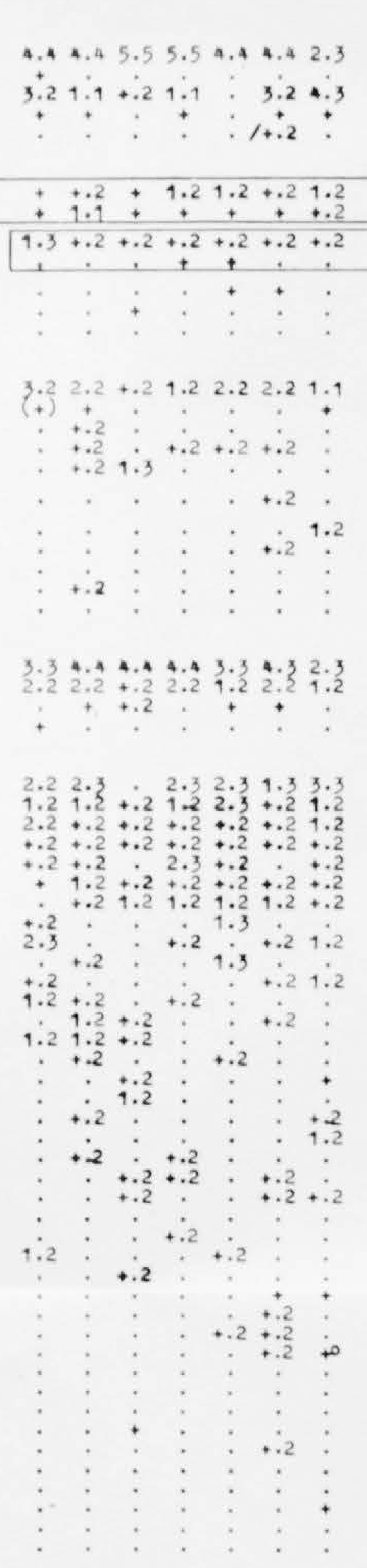 & 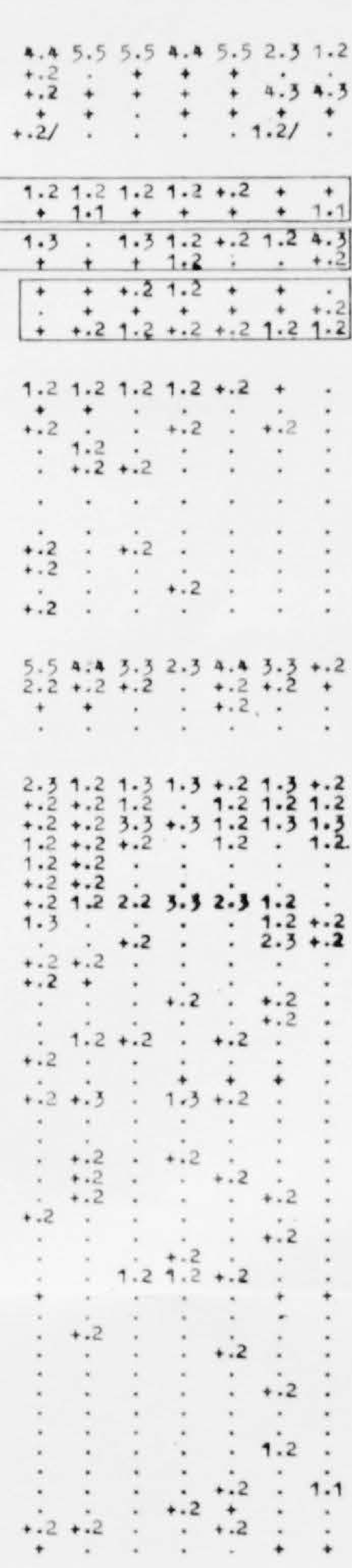 \\
\hline
\end{tabular}

Ausserdem in zwel Aufnahmen: Georgia pellucida: $1 \cdot 2(2),+\cdot 2(18) ;$ Polytrichum juniperium: $\boldsymbol{H}_{2}(3), 1 \cdot 2(24) ;$ Cladonia silvatica: $+\cdot 2(6,22) ;$ Festuca sp.: $+\cdot 2$ (6, 27): Cladonia coniocraea: $+\cdot 2(7,32)$; Rhacomitrium heterostichum: $+\cdot 2(9,13) ;$ Anastrepta orcadensis; +2 (10, 18); Rhytidiadetphus triquetrus: +2 (10, 27): Sphagnum recurvum: $2 \cdot 3(17,32)$; Potentilla aurea: 4 (23, 33); Cicerbita alpina: $+(27,28)$; Galium anisophyllum: $1 \cdot 2(31),+\cdot 2(33) ;$ Luzula pilosa: $+\cdot 2(31),+(33) ;$

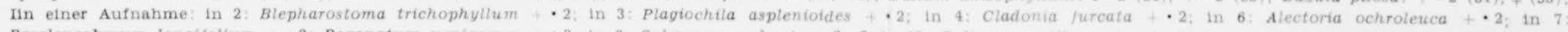
Paraleucobryum longifolium $+\cdot 2$; Pogonatum urnigerum $\cdot 2$ : in 8: Sphagnum robustum $2 \cdot 3 ;$ in 12 : Caltum saxatile $1:$ in 14 : Cystopteris filix-fragitis $+;$ in $15:$ Dryopteris austriaca ssp. spinulosa $+\cdot 2$; in 16 : Lecidea grannosa $+\cdot 2$; in 21 : Chitos cyphus polyouthus $+;$ in 22 ; Arctoa futvella + , Lophacotea heterophylta $+\cdot 2$; in 24 : Anemone alpina ssp. eu-alpina +, Hieracium murorum +, Rhacomitryum sudeticum + $\cdot 2$; in 26 ; Sphagnum sp. $\cdot 2$; in 29: Eptlobium angustijolium t. Dicranella heteromalla

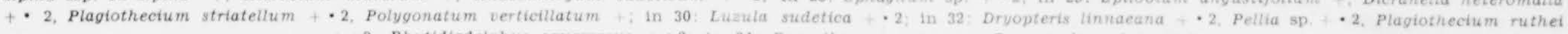
$\cdot 2$, Rhytidiadelphus squarrosus $\cdot 2 ;$ in 34 ; Prenathes purpurea : Ranunculus platani/olins 
Tabelle 8

Gesellschaftsvergleich der Knieholzgebüsche des Riesengebirges und der Karpathen

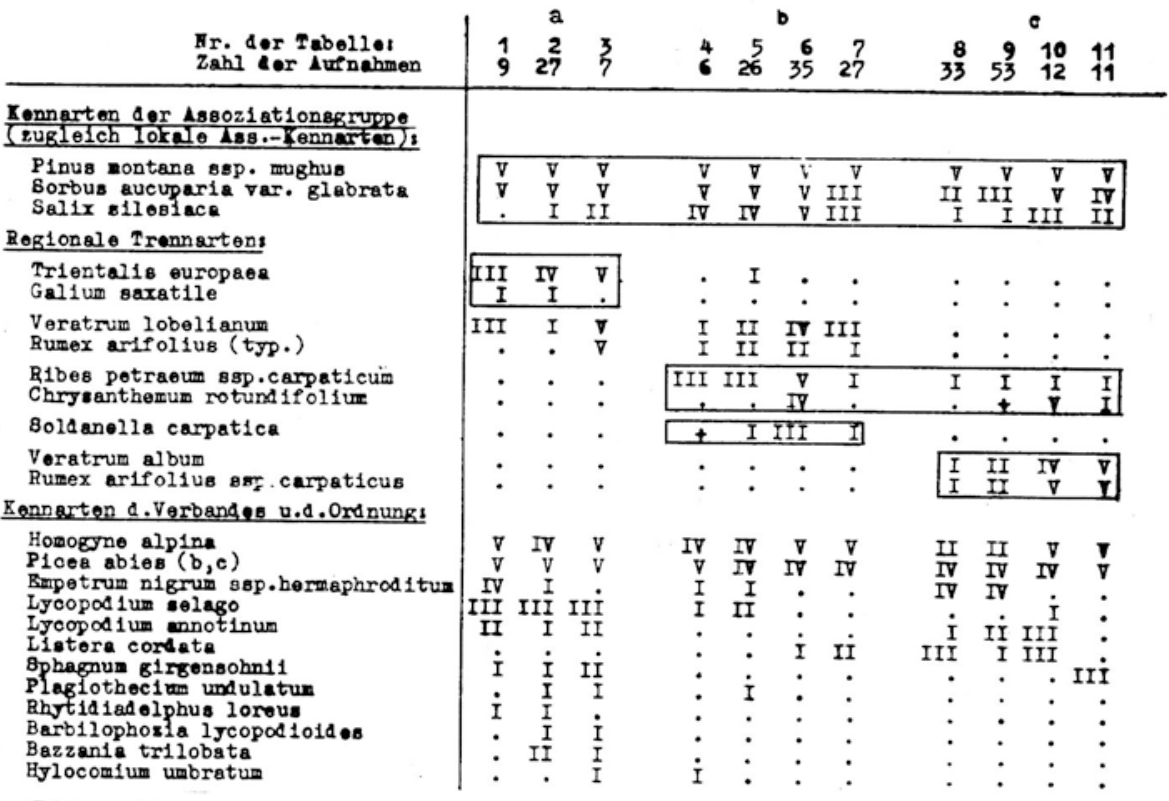

a - Riesengebirge

1. Pinetum montanae nach $\mathrm{K}$. $\mathrm{H} \mathrm{ueck}$ (1939, Tab. 9.)

2. Pinetum mughi sudeticum typicum (diese Arbeit, Tab. 7.)

3. Pinetum mughi sudeticum rumicetosum (diese Arbeit, Tab. 7.)

b - Westkarpathen

4. 6 Aufn. von W. Matuszk1ewicz (n.p.) aus dem Pilsko-Gebirge

5. 16 Aufn. von J. Walas (1933, Tab. XV.) und 10 Aufn. von W. Matuszkiewicz (n.p.) aus dem Babia Gora-Gebirge

6. Pinetum mughi carpaticum calcicolum nach B. Paw łow ski (zit. Pawowski B., W a 1 a s J., 1949, Tab. XVII.)

7. Pinetum mughi carpaticum silicicolum nach B. Paw łow $\mathrm{ski}$ (zit. P a w ło w ski B., W a 1 a s J., 1949, Tab. XVII.)

c - Ostkarpathen

8. Pinetum mughi carpaticum silicicolum aus den Gorganen ( $\mathrm{S}$ u $1 \mathrm{~m}$ a $\mathrm{T} .$, 1929, Tab. II.)

9. Pinetum montanum silicicolum aus den Gorganen ( $\mathrm{Tr}$ a m ple r T., 1937, Aufn. 9-61)

10. Mughetum carpaticum rumicetosum aus den Gorganen; nach den Aufnahmen von T. S u 1$\mathrm{ma}$ und T. Trampler zusammengestellt von B. Pawłowski und J. Walas (1949, Tab. XVII.)

11. Mughetum carpaticum rumicetosum aus der Czarnohora und aus dem Czywczyn-Gebirge (P a w 0 w sk1 B., W a 1 a s J., 1949, Tab. XVII.)

2. Beide Gesellschaften lassen sich gut durch regionale Trennarten unterscheiden. Im Riesengebirge sind es boreal-kontinentale Trientalis europaea und subatlantisches Galium saxatile; in den Karpathen - subendemische Sippen Ribes petraeum ssp. carpaticum, Chrysanthemum, rotundifolium, Soldanella carpatica (Westkarpathen) und Rumex arifolius ssp. carpaticus (Ostkarpathen) nebst einigen weniger bezeichnenden Begleitern. 
3. Manche Arten, welche in den Karpathen als Kennarten der Legföhrengebüsche gelten, haben im Riesengebirge entschieden andere soziologische Bindung. So sind z. B. Athyrium alpestre, Betula carpatica, Padus avium ssp. petraea im unseren Gebiet bestimmt als Betulo-Adenostyletea - Arten aufzufassen, die im Knieholz höchstens in gewissen, zu den Hochstaudenfluren übermittelnden Ausbildungen vorkommen und dort als deren Trennarten angesehen werden können. Andere Arten, welche wie Rosa pendulina, Streptopus amplexifolius, Dryopteris austriaca ssp. dilatata $u$. a. aus der subalpinen Stufe der Karpathen als Vaccinio-Piceion - Arten angegeben wurden, müssen im Riesengebirge zu den Begleitern gestellt werden.

4. Die Legföhrengesellschaften der Westkarpathen und des Riesengebirges unterscheiden sich auch in der Art der Untergliederung. Dort hat sich die grundsätzlich petrographisch und topographisch bedingte Einteilung in "calcicolum" und „silicicolum" gut bewährt; hier wollen wir die Gesellschaft eher nach dem Wasserhaushalt in die Subassoziationen „typicum" und „rumicetosum" unterteilen.

Die angeführten Tatsachen zeigen darauf hin, dass die Gesellschaften des Knieholzes in den Karpathen und im Riesengebirge verschiedene, wenn auch verwandte, Artenverbindungen aufweisen und so den Rang paralleler, sich ersetzender Gebietsasscziationen verdienen. Sie sind $u$. E. in demselben Masse voneinander verschieden, wie die Fichtenwälder der betreffenden Gebiete, deren systematische Trennung ja nicht angefechtet wird.

Lokal wird die Gesellschaft besonders durch die charakteristischen Sträucher gekennzeichnet und gegen das Piceetum abgegrenzt. Es gibt auch einige Verbandsarten, welche ohne im Pinetum mughi vollkommen zu fehlen, doch ihren Schwerpunkt im Fichtenwald haben; so z. B. die Moose Plagiothecium undulatum, Hylocomium umbratum und Rhytidiadelphus loreus.

Pinetum mughi sudeticum zeigt in den Höhenlagen zwischen 1250 und $1450 \mathrm{~m}$ ü. d. M. keine Abhängigkeit von den topographischen und Bodenfaktoren, soweit die Assoziation als Ganzes betrachtet wird (s. Fig. 13.). Sie ist für diese Stufe als Vegetationsklimax aufzufassen. In tieferen Lagen kommt die Gesellschaft extrazonal an besonders zusagenden Standorten auch innerhalb der Waldstufe vor. Das ist vor allem bei den Frostlöchern der Kare der Fall; auch steigt die Gesellschaft auf nordexponierten, blockreichen und lavinenzügigen steilen Hängen sehr tief́ hinab. Oberhalb etwa $1450 \mathrm{~m}$ ü. d. M. gibt es meistens nur kleinflächige, fragmentarisch entwickelte Bestände, welche am Nordabhang der Schneekoppe gegen Melzergrund bei rund $1500 \mathrm{~m}$ ü. d. M. ausklingen. 


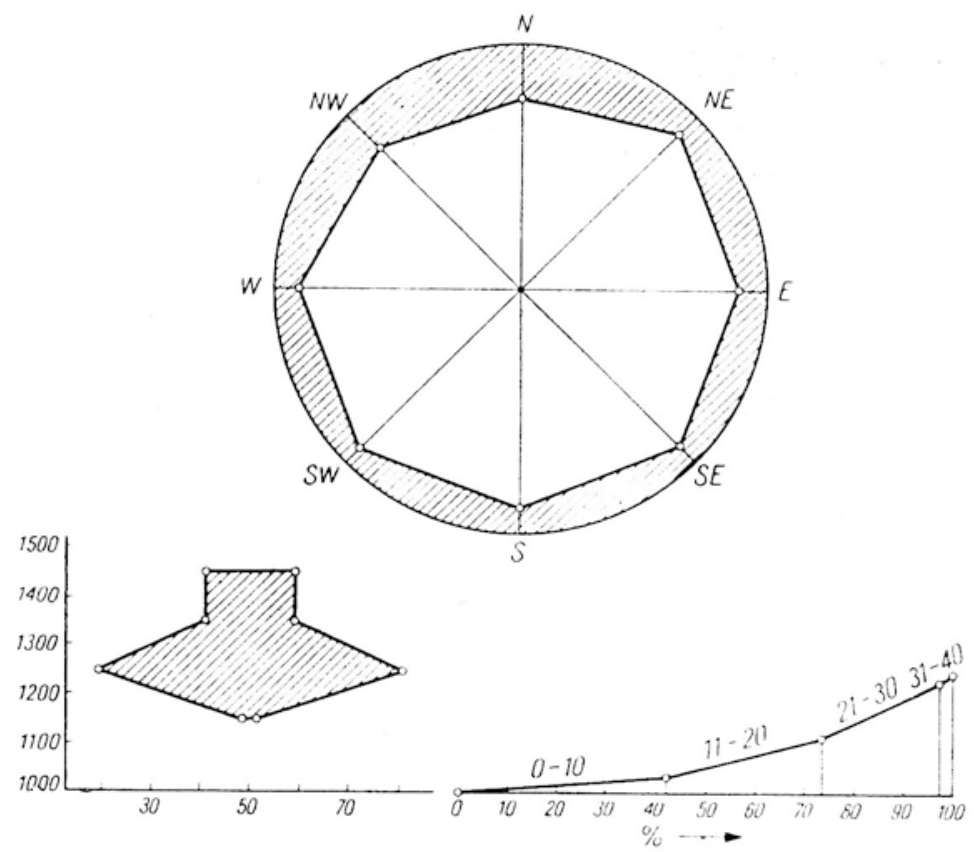

Fig. 13. Verteilung der Aufnahmen des Pinetum mughi sudeticum im Riesengebirge auf die verschiedenen Himmelsrichtungen, Bodenneigungen und Höhenlagen

Die häufigsten Böden sind es im Pinetum mughi feinerdearme, stark sauere oligotrophe Rankern mit ein€r mächtigen Rohhumusauflage (s. Fig. 14.). Es gibt aber auch Bestände die auf primären Podsolen oder auf anmoorigen Böden stocken. Die Unterschiede der Wasser- und Bodenverhältnissen kommen in den Ausbildungsformen der Gesellschaft zum Ausdruck.

Pinetum mughi sudeticum lässt sich folgendermassen untergliedern:

A. Pinetum mughi typicum - typische Subassoziation ohne Differentialarten.

a. R e in e V a riante stellt eine relativ trockenere Ausbildung der Gesellschaft dar. Besonders bezeichnend und für die Assoziation tatsächlich "typisch" ist die Homogyne-Subvariante mit Homogyne alpina und Trientalis europaea als Trennarten, während die r e in e S u bvarian t e eher als standortsbedingte Verarmungsform aufzufassen ist. Sie ist artenarm und schlechtwüchsig und auf felsblockreiche, windexponierte und schneearme Lagen beschränkt. Diese Einheit ist besonders für die Endmoränen der Kare recht charakteristisch.

b. Die Athyrium alpestre-Variante ist etwas artenreicher, was auf die Bodenfrische und Schneeschutzlage zurückzuführen ist. Mit 

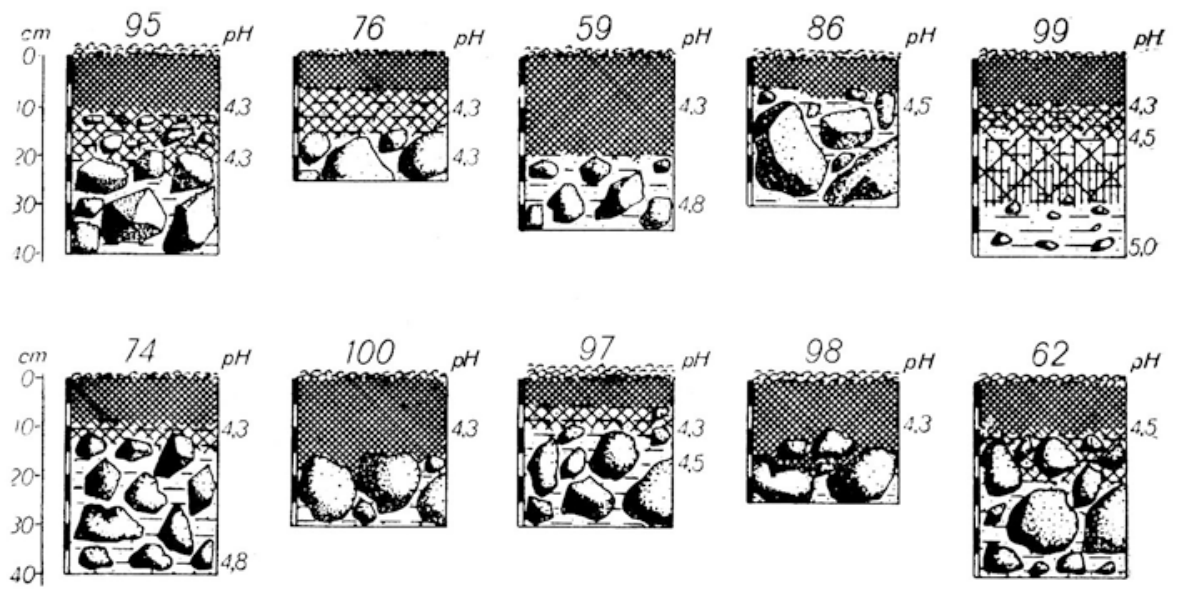

Fig. 14. Bodenprofile aus dem Pinetum mughi sudeticum des Riesengebirges

spärlich eingesprengten Betulo-Adenostyletea-Arten vermittelt die Gesellschaft einen Übergang zur hochstaudenreichen Subassoziation.

B. Pinetum mughi rumicetosum wird durch die aus den Kontaktge-sellschaften übergreifenden Betulo-Adenostyletea-Arten gut gekennzeichnet. Athyrium alpestre, Rumex arifolius und Veratrum lobelianum sind hochstet; Adenostyles alliariae, Senecio nemorensis u. a. kommen nicht selten vor. In der Strauchschicht fällt ein grösserer Anteil von Laubholzarten besonders auf. Die Gesellschaft ist auf gut durchfeuchtete Böden mit länger dauerndem Schneeschutz beschränkt und stellt sowohl floristisch, wie ökologisch die reichste Form der Assoziation dar, wenn auch deren Charakterzüge durch Anwesenheit gesellschaftsfremder Arten etwas beeinträchtigt werden. Die Subassoziation ist besonders gegen die obere Knieholzgrenze recht häufig, wo sie auch im kleinflächigen Wechsel mit den Hochstauden- und Grasfluren vorzukommen pflegt.

Ausser den genannten Einheiten gibt es im Riesengebirge Knieholzbestände auf tiefgründigen Torfböden. Ihre Beziehungen $\mathrm{zu}$ den Hochmoorgesellschaften sowie ihre systematische Fassung bedürfen noch einer Klärung.

Klasse: QUERCO-FAGETEA B r. - B l. et V li e g. 1937.

Ordnung: FAGETALIA SILVATICAE P a w 1. 1928.

Verband: FAGION Tx. et D i e m. 1936.

Unterverband: LUZULO-FAGION Loh m. et Tx. 1954.

Assoziation: LUZULO-FAGETUM MONTANUM O berdorfer 1950.

Der bodensauere Hainsimsen-Buchenwald kommt im Riesengebirge in der unteren montanen Stufe bis zu etwa $1000 \mathrm{~m}$ ü. d. M. vor. Er ist 


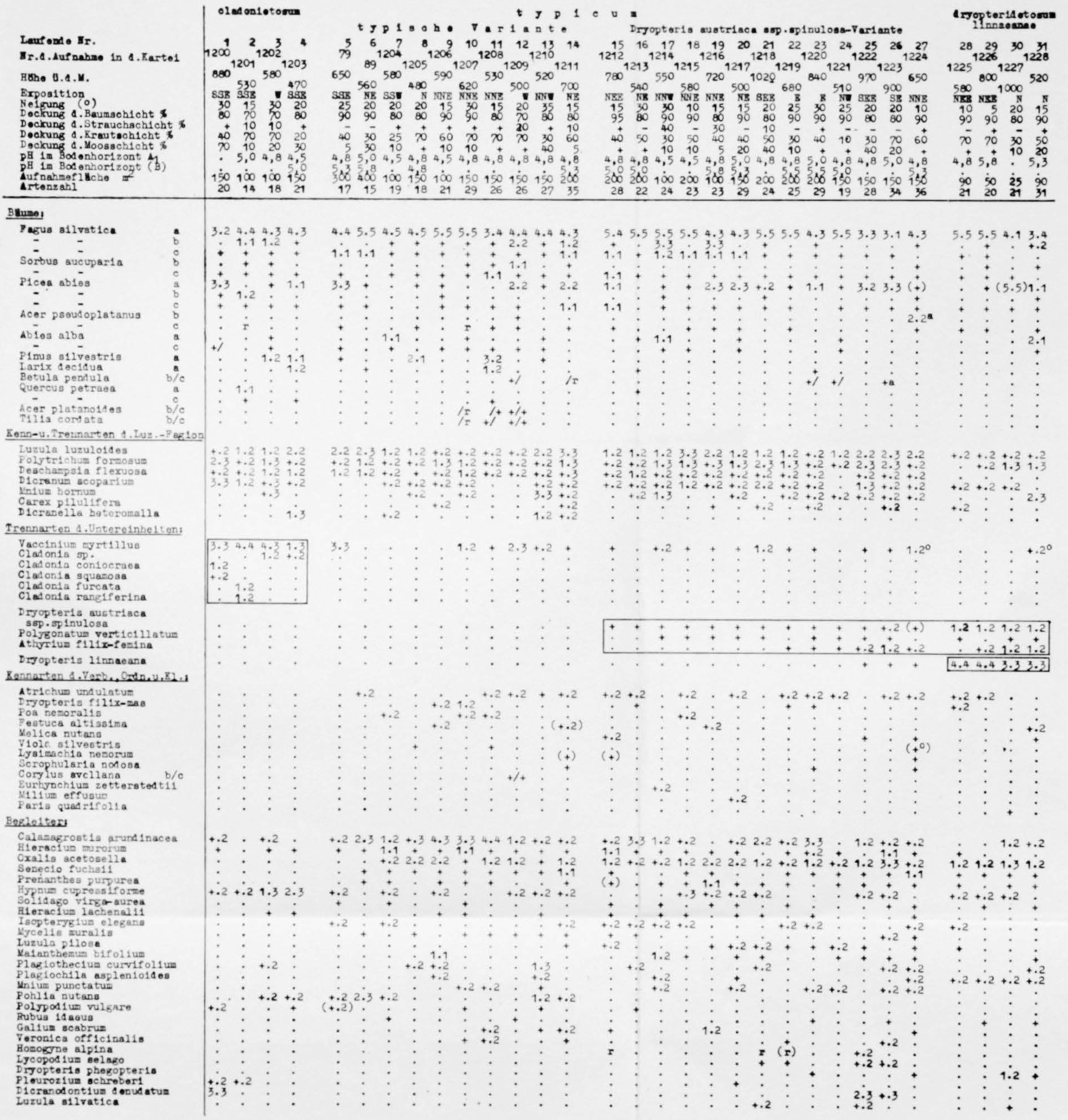

Ausserdem in zwel Aufnahmen: Bazzania trilobata: $+\cdot 2(1,20)$ : Dolichotheca silesiaca: +2 (6, 29); Galeopsis pubescens: $+(8,16) ;$ Epilobium angustifolium: $(12,14)$; Sambucus racemosa b: $+(31)$, c: r $(12) ;$ Cladonia sp : $+\cdot 2(13,16) ;$ Deschampsia caespitosa: $+\cdot 2(15),+(27) ;$ Isothecium myurum: $+\cdot 2$ (19, 28); Ca. Lamagrostis villosa: + $\cdot 2(19),+(29) ;$ Rubus sp : + $(23,29)$; Blechnum spicant: $\mathrm{r}(25),+\cdot 2$ (26). In elner Aufnahme: in 1: Paraleucobryum longifolium + $\cdot 2$, Vaccinium vitis-idea $\mathrm{r}$; in 4 : Orthodicranum montanum $+\cdot 2$, Rhabdoweissia fugax $+\cdot 2 ;$ in 10 : Campanula rotundifolia + , Galium mollugo + . Plagio thecium denticulatum $+\cdot 2$; in 12: Fragaria vesca $r$, Platanthera bifolia + , Quercus robur c $r$; in $14:$ Digitalis purpurea $\mathrm{r}$, Ditrichum homomalum $+\cdot 2$, Luzulo multiflora + , Pogonatum aloides $+\cdot 2$, Polytrichum juniperinum $+\cdot 2$; In 15 : Angelica silvestris $(+)$, Pyrola minor $(+)$; in 17: Diplophyllum albicans $+\cdot 2 ;$ in 20 Galeopsis speciosa $+;$ in 21: Lophocolea heterophylla $+\cdot 2$, Rhytidiadelphus triquetrus $+\cdot 2 ;$ in $25:$ Calypogeia trichomanis $+\cdot 2$, Gentiana asclepiadea $+;$ in 26. Myosotis scorpioides ssp. palustris +, Ptilidium ciliare + $\cdot 2$; in 27 : Ajuga reptans +, Rubus sect, glandulosi +i in 30 : Adenostyles alliariae + , Mnium spino. sum $+\cdot 2 ;$ in 31 Circaea alpina + Epilobium montanum,+ Pellia sp. $+\cdot 2$, Plagiothectum undulatum $+\cdot 2$, Pogonatum urnigerum $+\cdot 2$, Stellaria nemorum $+;$ 
zwar durch einseitige Fichtenwirtschaft stark zurückgedrängt worden, doch gibt es noch gut entwickelte Bestände (so z. B. auf dem Kynast und um den Kochelfall), welche das Gefüge der Gesellschaft erkennen lassen.

Die azidophilen Buchenwälder der montanen Stufe, welche im westlichen Mitteleuropa schon recht gut durchgeforscht sind, wurden von unseren Pflanzensoziologen bisher ausserachtgelassen. Als erster hat S. M y c z k ow ski (1958) einige diesbezüglichen Aufnahmen veröffentlicht und sich gewagt diese Einheit nach Vorbild deutscher Forscher als Luzulo-Fagetum auszuscheiden, ohne allerdings auf die Frage nach der systematischen Stellung der Gessellschaft einzugehen. Nachdem wir uns die Hainsimsen-Buchenwälder in Ost- und Westdeutschland angesehen und einigermassen kennen gelernt haben besteht für uns kein Zweifel mehr dass hier eine besondere Gruppe von Fagetalia-Gesellschaften

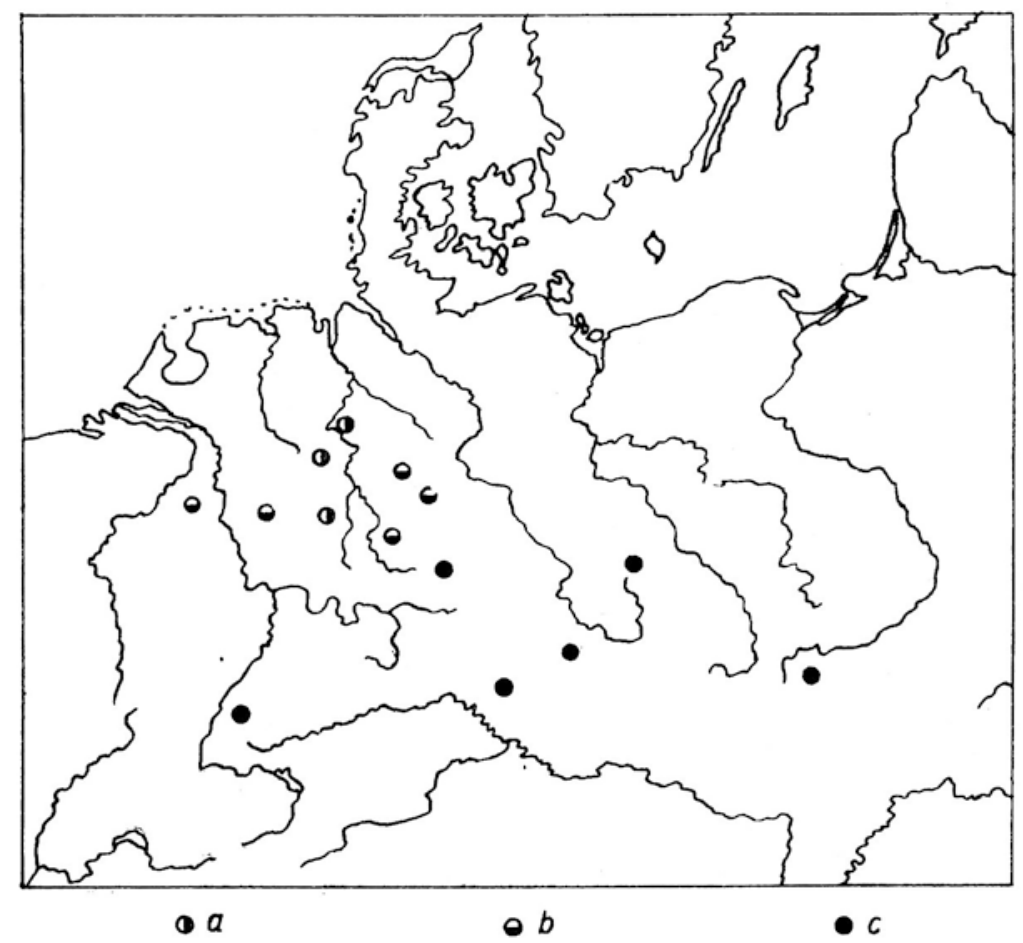

Fig. 15. Regionale Verteilung der auf Grund von Tabelle 10. ausgeschiedenen Einheiten des Hainsimsen-Buchenwaldes in Mitteleuropa a - Luzulo-Fagetum hercynicum, reine Ausbildung der tieferen Lagen; b - Luzulo-Fagetum hercynicum, Polygonatum verticillatum-Ausbildung der mcntanen Stufe; c - Luzulo-Fagetum 
vorliegt. Wir haben sie, der Auffassung von E. O be rd orfer (1957) folgend, als einen Unterverband dem Fagion angeschlossen.

Wir haben unsere Gesellschaft mit einigen in der jüngsten Zeit aus Mitteleuropa bekanntgewordenen Luzulo-Fageten verglichen, ohne allerdings die Frage nach der regionalen Gliederung dieser Gesellschaftsgruppe lösen zu wollen, was ja ohne eingehende Vergleichsuntersuchung des gesamten verfügbaren Materials gar nicht möglich wäre. Dennoch ergeben sich schon beim vorläufigen Vergleich recht interessante Zusammenhänge, welche wenigstens als Arbeitshypothese eine Erwähnung verdienen. (s. Tab. 10.). Sie lassen sich kurz wie folgt zusammenfassen:

1. Die verglichenen Luzulo-Fageten sind wahrscheinlich in zwei Gebietsassoziationen aufzugliedern, nämlich eine nordwesteuropäische und eine südmitteleuropäisch-montane (Fig. 15.). Die letztere wird durch die montanen Arten: Abies alba, Prenanthes purpurea, Senecio fuchsii u. a. gekennzeichnet; sie umfasst die azidophilen Buchenwälder des Schwarzwaldes, des Bayerischen Waldes, des Thüringischen Schiefergebirges, der Sudeten, böhmischer Gebirgen und Westkarpathen. Diese Einheit sollte etwa dem Luzulo-Fagetum montanum Oberdorfer 1950 entsprechen.

2. Die nordwesteuropäische Gesellschaft, welche wir vorläufig Luzulo-Fagetum hercynicum Tx. 1954 nennen wollen, scheint in zwei Höhenausbildungen vorzukommen, welche durch einen verschieden starken Anteil von montanen Arten unterschieden werden.

3. Unsere lokale Ausbildung aus dem Riesengebirge zeigt mit $\mathrm{Ga}$ lium saxatile und Digitalis purpurea (subspontan) höchste Verwandtschaft mit den Luzula-Buchenwäldern anderer Sudeten-Züge, sowie des Thüringischen Schiefergebirges und des Böhmischen Mittelgebirges. Das Luzulo-Fagetum, welches uns S. M y c zkow ski (1958) aus Westbeskiden mitgeteilt hat, weicht dagegen in seinem floristischen Gefüge etwas ab so, dass es hier vielleicht mit einer besonderen geographischen Rasse $\mathrm{zu}$ rechnen ist.

An Standortsformen können im Riesengebirge folgende Untereinheiten ausgeschieden werden, die mit denjenigen aus Westdeutschland weitgehend übereinstimmen.

A. Luzulo-Fagetum cladonietosum, durch die Cladonien und massenhaftes Auftreten der Heidelbeere ausgezeichnet, stellt den trockensten, ,linken" Flügel der Assoziation dar. Die Gesellschaft ist auf die süd- und ostexponierten, durch Wind ausgehagerten Hänge in tieferen Lagen beschränkt. Sie kommt im Gebiete nur auf konvexen Berghängen isolierter Kuppen, so z. B. auf dem Kynast und dem Herdeberg vor. 
T a be 11 e 10

Gesellschaftsvergleich einiger Hainsimsen-Buchenwälder Mitteleuropas

\begin{tabular}{|c|c|c|c|c|c|c|c|c|c|c|c|c|c|c|}
\hline \multirow{2}{*}{$\begin{array}{l}\text { Nr.der. Tabelle } \\
\text { Zahl der Aufnahmen } \\
\text { W1ttlere Artenzehl }\end{array}$} & \multicolumn{4}{|c|}{ a } & & \multicolumn{4}{|c|}{ c } \\
\hline & $\begin{array}{rr}1 & 2 \\
3 & 6 \\
13 & 13\end{array}$ & $\begin{array}{r}3 \\
10 \\
10\end{array}$ & $\begin{array}{r}4 \\
11 \\
12\end{array}$ & $\begin{array}{r}5 \\
14 \\
.\end{array}$ & $\begin{array}{r}6 \\
6 \\
17\end{array}$ & $\begin{array}{r}7 \\
16 \\
13\end{array}$ & $\begin{array}{r}8 \\
32 \\
10\end{array}$ & $\begin{array}{r}9 \\
11 \\
16\end{array}$ & $\begin{array}{l}10 \\
12 \\
13\end{array}$ & $\begin{array}{l}11 \\
13 \\
22\end{array}$ & $\begin{array}{l}12 \\
17 \\
14\end{array}$ & $\begin{array}{l}13 \\
23 \\
25\end{array}$ & $\begin{array}{r}14 \\
9 \\
.\end{array}$ & $\begin{array}{rr}15 & 16 \\
14 & 15 \\
\cdot & 24\end{array}$ \\
\hline \multicolumn{15}{|l|}{ Xonn-u.Trannarton a.Iuz.-Fagion } \\
\hline $\begin{array}{l}\text { Luzula luzuloides } \\
\text { Polytrichum formosum } \\
\text { Deschampia flexuosa } \\
\text { Dicramelia heteromalla } \\
\text { Vaccinium myrtillus } \\
\text { Carex pilulifera } \\
\text { Dicranum scoparium } \\
\text { unium hornum }\end{array}$ & \begin{tabular}{|rr}
3 & V \\
$\vdots$ & III \\
3 & III \\
$i$ & II \\
1 & III \\
1 & $\ddots$
\end{tabular} & $\begin{array}{r}V \\
V \\
\nabla \\
I \\
\text { III } \\
V \\
\text { III } \\
.\end{array}$ & $\begin{array}{r}\mathrm{V} \\
\mathrm{V} \\
\mathrm{IV} \\
\mathrm{II} \\
\dot{\mathrm{V}} \\
\mathrm{II} \\
\end{array}$ & $\begin{array}{l}\text { V } \\
\text { IV } \\
\text { III } \\
\text { III } \\
\dot{I} \\
\dot{\text { II }}\end{array}$ & $\begin{array}{c}\nabla \\
\nabla \\
V \\
+ \\
I V \\
V \\
+ \\
+\end{array}$ & $\begin{array}{r}\text { V } \\
\text { III } \\
\text { II } \\
\text { II } \\
\text { I } \\
\vdots \\
\text { I }\end{array}$ & $\begin{array}{c}\text { V } \\
\text { IV } \\
\text { I } \\
\text { I } \\
\vdots \\
\end{array}$ & $\begin{array}{r}V \\
\text { IV } \\
\nabla \\
I \\
\text { III } \\
\dot{I I} \\
\dot{I I}\end{array}$ & $\begin{array}{l}\text { V } \\
\text { III } \\
V \\
\text { III } \\
\text { IIII } \\
\text { III } \\
\underset{I}{I}\end{array}$ & $\begin{array}{r}\mathrm{V} \\
\mathrm{II} \\
\mathrm{III} \\
\dot{I} \\
\mathrm{I} \\
.\end{array}$ & $\begin{array}{r}\text { III } \\
\text { IV } \\
V \\
I \\
V \\
I \\
I \\
I I \\
I \\
I\end{array}$ & $\begin{array}{r}\text { V } \\
V \\
V \\
I \\
I V \\
I I \\
I V \\
\text { III }\end{array}$ & $\begin{array}{c}\text { V } \\
\text { IV } \\
\text { V } \\
\text { I } \\
\text { I } \\
\text { II } \\
.\end{array}$ & $\begin{array}{rrr}\text { V } & \text { V } \\
\text { II } & \text { III } \\
\text { IV } & \text { II } \\
\text { II } & \text { I } \\
: & \text { I } \\
: & + \\
& \end{array}$ \\
\hline \multicolumn{15}{|l|}{ Reglonale Trennarten: } \\
\hline $\begin{array}{l}\text { Sombucus racemosa } \\
\text { Polygonatum verticillatur }\end{array}$ & : & : & : & & $\begin{array}{l}\mathrm{I} \\
\mathrm{I}\end{array}$ & II & I & I & IV & II & II & $I I$ & I & $\begin{array}{lr}I & I \\
I & I I \\
\end{array}$ \\
\hline $\begin{array}{l}\text { Ables alba } \\
\text { Senecio fuchsii } \\
\text { Prenanthes purpurea }\end{array}$ & $: \quad:$ & I & $\begin{array}{l}I \\
:\end{array}$ & : & $\dot{0}$ & $\dot{.}$ & : & : & : & & $\begin{array}{l}\text { I } \\
\text { I } \\
\text { I }\end{array}$ & $\begin{array}{l}\text { III } \\
\text { IV } \\
\text { IV }\end{array}$ & II & $\begin{array}{ll}V & V \\
\text { II } & \text { III } \\
\text { IV } & \text { II } \\
\end{array}$ \\
\hline
\end{tabular}

a - Wesergebirge ( $\mathrm{L}$ o h m e y e $\mathrm{r}$ W., T ủx en R., 1958)

1. Luzulo-Fagetum

Schlitzerbergland (S e i b e r t P., 1954)

2. Dryopteris linnaeana-Ausbildung

3. reine Ausbildung

4. Oxalis-Ausbildung

\} reiner Luzula-Buchenwald

Eggegebirge ( $T r$ a t mann W., 1957)

5. Luzulo-Fagetum

b - Ardennen (Noirfalis e A., 1949)

6. la hêtraie à Luzula nemorosa

NW Harz ( $\mathrm{T} \ddot{\mathrm{u}} \mathrm{x}$ e n R., 1954)

7. Luzulo-Fagetum hercynicum dryopteridetosum linnaeanae

8. Luzulo-Fagetum hercynicum typicum

Unterharz - Kyffhäuser

9. Luzulo-Fagetum (Aufn. 10-18 aus der Tab. I. von Meusel H. 1954 und 2 Aufn. von W. Matuszkiewicz - n.p. - )

Ruhlaer Gebirge, NW Thüringer Wald (S chlüter H., 1959)

10. Luzulo-Fagetum typicum

11. Luzulo-Fagetum milietosum

c - Thüringisches Schiefergebirge (Grüneberg H., S c h l ü t e r H., 1957)

12. Luzulo-Fagetum, typische Variante und Waldreitgrasvariante

Riesengebirge (diese Arbeit, Tab. 9)

13. Luzulo-Fagetum montanum typicum

Schwarzwald (O berdorfer E., 1957)

14. Luzulo-Fagetum montanum typicum

15. Luzulo-Fagetum montanum luzuletosum silvaticae

Westbeskiden (M y c z k o w s 1 S., 1958)

16. Luzulo-Fagetum, typische Variante

B. Luzulo-Fagetum typicum - ohne eigene Trennarten - stellt die normale und verbreitetste Form der Gesellschaft dar. Dem Wasserhaushalt gemäss kann man zwei Varianten unterscheiden:

a. Reine Variante ist etwas trockener und daher in tieferen Lagen, besonders auf konvexen, offenen Hängen häufiger. 
b. Die Dryopteris-Vari a n te mit Dryopteris austriaca ssp. spinulosa, Polygonatum verticillatum und Arthyrium filix-femina als Trennarten, bevorzugt frischere Standorte und ist daher in höheren Lagen, oder auf konkaven, durch den Gegenhang geschützten Hängen öfter anzutreffen. Sie ist im Gebiete relativ verbreitet und stellt eigentlich „,typische" Form der Assoziation dar.

C. Luzulo-Fagetum dryopteridetosum linnaeanae wird in seinem Aspekt durch den Farnreichtum bestimmt. Ausser der namengebenden Art, welche meist hohen Deckungsgrad erreicht, kommen hier andere Farne, so vor allem Athyrium filix-femina, Dryopteris austriaca (beide Subspezies) und Dryopteris filix-mas, häufiger und zahlreicher als in übrigen Einheiten der Gesellschaft vor. Die Subassoziation ist streng an die Nordlagen und kleinere, bodenfrische bis -feuchte Mulden gebunden und ist, vom forstwirtschaftlichen Standpunkt aus betrachtet, als die leistungsfähigste Form des Hainsimsen-Buchenwaldes zu bezeichnen.

Die Abhängigkeit des Luzulo-Fagetum von den topographischen Faktoren ist aus der Fig. 16. zu sehen. Im Hauptbereich seiner vertikalen

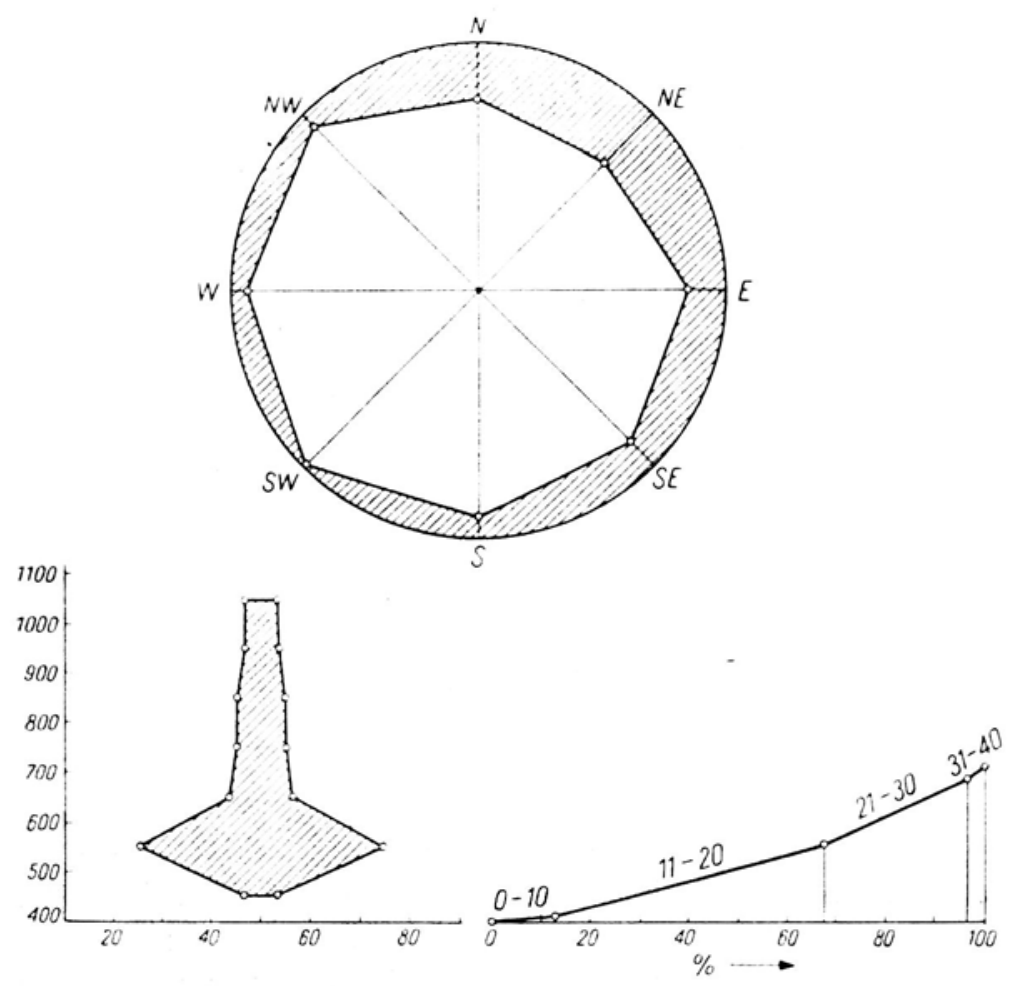

Fig. 16. Verteilung der Aufnahmen des Luzulo-Fagetum montanum im Riesengebirge auf die verschiedenen Himmelsrichtungen, Bodenneigungen und Höhenlagen 


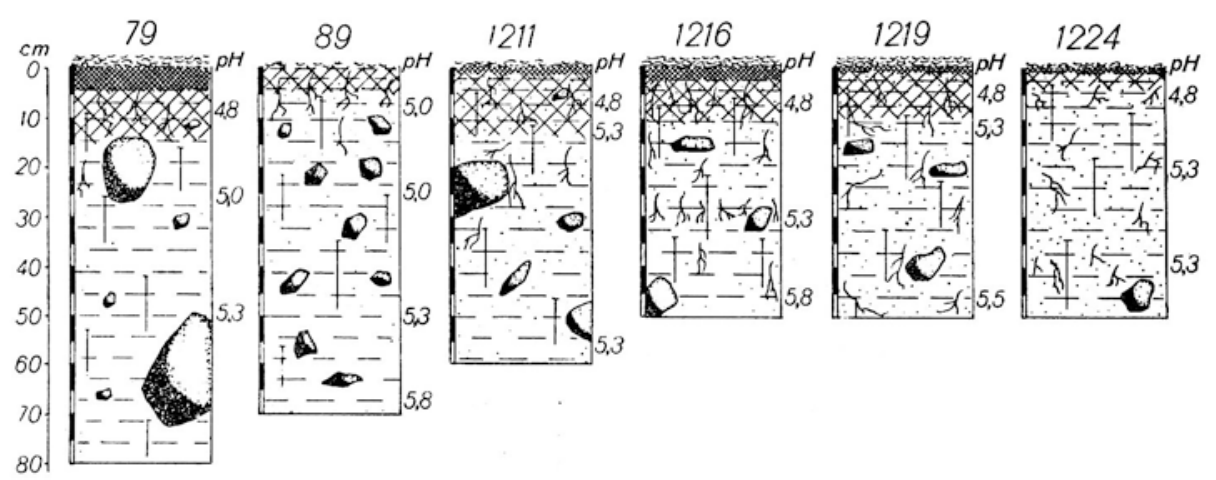

Fig. 17. Bodenprofile aus dem Luzulo-Fagetum typicum des Riesengebirges

Verbreitung lässt sich keine Bindung an bestimmte Geländeformen nachweisen. In etwas höheren Lagen ( $\pm 800-1000 \mathrm{~m}$ ü. d. M.) meidet die Gesellschaft alle frostgiefährdeten Standorte, wie z. B. tiefe Bachtäler, abflusslose Mulden und Becken udg. Obwohl Mehrzahl der Aufnahmen aus den Höhenlagen zwischen $500-600 \mathrm{~m}$ ü. d. M. stammt, was auf verschieden starke Beeinflussung durch forstwirtschaftliche Massnahmen zurückzuführen ist, doch lässt sich auf Grund eingestreuter Fragmente und einzelner Horste feststellen, dass das Luzulo-Fagetum in der unteren montanen Stufe allgemein zu Hause ist. Es ist sogar anzunehmen, dass gerade der Hainsimsen-Buchenwald als Vegetationsklimax für diese Stufe aufzufassen ist.

Die Böden unserer Gesellschaft sind als oligotrophe, schwach podsolierte und recht sauere Braunerden zu bezeichnen (s. Fig. 17). Sie sind skelettreich und meist mittelgründig; ihre Humusform ist Moder; sie sind auch biologisch wenig aktiv.

Unterverband: EU-FAGION Oberdorfer 1957.

Assoziation: DENTARIO ENNEAPHYLLIDIS-FAGETUM Oberd. 1947.

Der artenreiche Buchenwald ist im Untersuchungsgebiet viel seltener als das Luzulo-Fagetum und kommt meist in kleinen, örtlich begrenzten Beständen vor. Auf Grund dreier in der Tabelle 11. zusammengestellten Aufnahmen wäre es kaum möglich systematische Stellung der Gesellschaft $\mathrm{zu}$ bestimmen, ebensowenig ihre ökologische Differenzierung zu erfassen. Wir verfügen jedoch über ein ziemlich umfangreiches Aufnahmematerial aus dem ganzen Gebiet der schlesischen Sudeten, welches uns erlaubt hat jene Fragen einigermassen geklärt zu haben. Auf die Ergebnisse dieser 
Tabelle 11

Dentario enneaphyllidis-Fagetum $\mathrm{Ob}$ e r d orfer

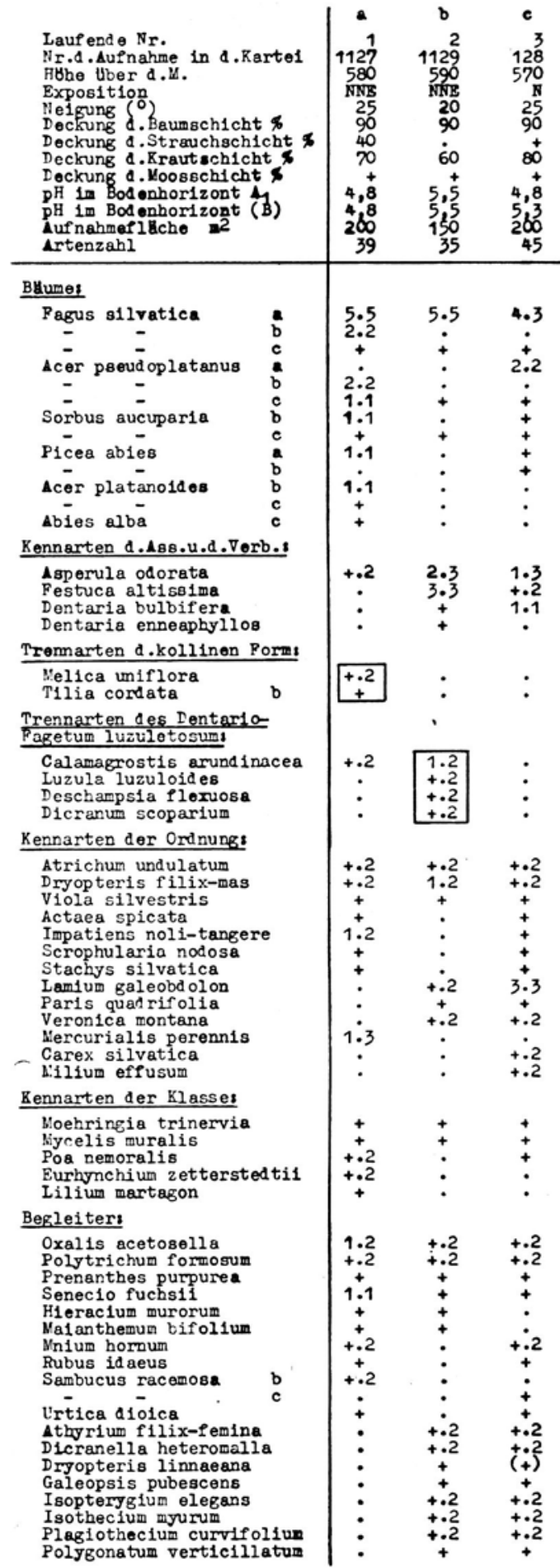


noch zu veröffentlichenden Bearbeitung wollen wir uns im vorliegenden Aufsatz stützen, ohne die betreffenden Fragen gründlich erörtern zu wollen.

Der artenreiche Buchenwald der Sudeten gehört einer besonderen Gebietsassoziation an, welche mit keiner anderen unserer Buchenwaldgesellschaften $\mathrm{zu}$ identifizieren ist. Insbesondere darf er weder dem montanen Fagetum carpaticum noch dem Melico-Fagetum der Moränengebiete angeschlossen werden. Er stellt grundsätzlich dieselbe Artenkombination dar, wie die analogen Gesellschaften des Böhmischen Mittelgebirges und des Erzgebirges (Preis K., 1938) sowie des Bayerischen Waldes ( $\mathrm{Trautm}$ an $\mathrm{n}$ W., 1952). Als regionale Assoziationskennarten können wir Asperula odorata, Dentaria bulbifera, Dentaria enneaphyllos und vielleicht auch Actaea spicata betrachten. Gegen das Melico-Fagetum wird die Gesellschaft durch mächtigen Anteil montaner Arten, gegen das Fagetum carpaticum - durch Fehlen seiner meist subendemischen Kennarten sowie eine abweichende Art der Untergliederung ausreichend abgegrenzt. Auch hat die kennzeichnende Art Dentaria enneaphyllos regional betrachtet ihren Schwerpunkt entschieden in unserer Assoziation, wenn sie auch in den westlichsten Arealteil von Fagetum carpaticum eindringt.
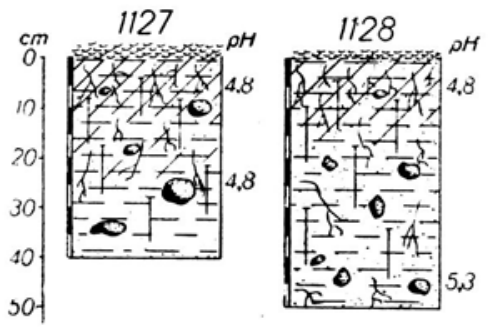

Fig. 18. Bodenprofile aus dem Dentario enneaphyllidis-Fagetum des Riesengebirges

Dentario enneaphyllidis-Fagetum der Sudeten lässt sich in eine Reihe von standortsbedingten Untereinheiten aufgliedern; es ist auch zwischen einer submontan-kollinen und einer montanen Form zu unterscheiden. Daraus ergibt sich ein recht buntes Bild der inneren Variabilität der

Fortsetzung der Tabelle 11:

Ausserdem in einer Aufnahme: in 1: Chaerophyllum temulum + , Galium scabrum + , Geranium robertianum ${ }_{+}$, Geum urbanum ${ }_{+}$, Lonicera nigra $\mathrm{c}_{+}$, Mnium affine $+\cdot 2$; in 2 : Solidago virga-aurea + , Veronica officinalis $+;$ in $3:$ Brachythecium velutinum $+\cdot 2$, Dryopteris austriaca ssp. spinulosa $+\cdot 2$, Equisetum silvaticum + , Luzula pilosa $(+\cdot 2)$, Minium punctatum $+\cdot 2$, Plagiochila asplenioides $+\cdot 2$. 


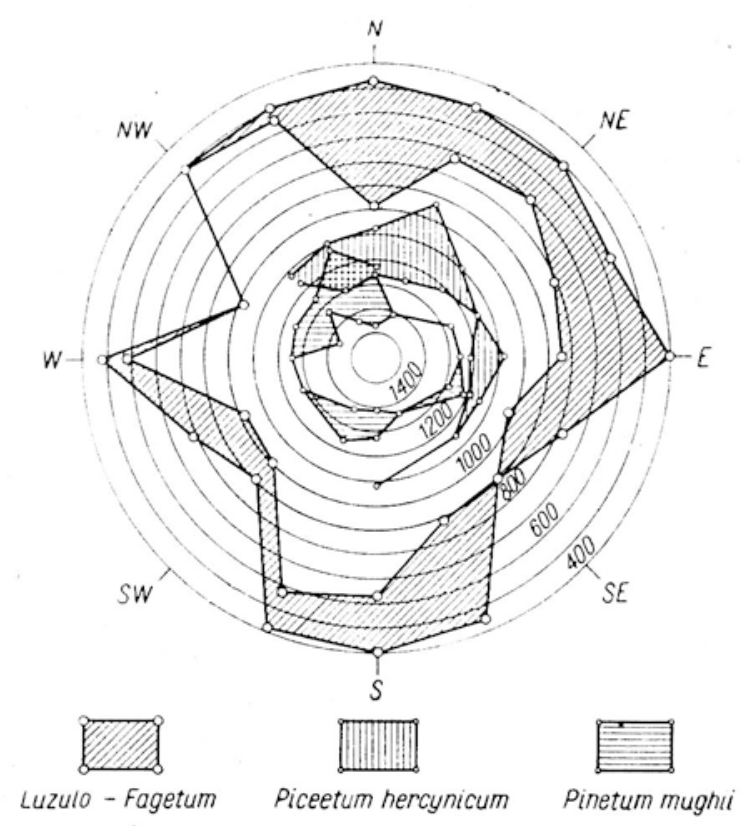

Fig. 19. Beziehung zwischen Exposition und Höhenlage der wichtigsten Waldgesellschaften des Riesengebirges auf Grund der Vegetationsaufnahmen

Assoziation. Drei in der Tabelle 11. zusammengestellte Aufnahmen stellen drei verschiedene Einheiten dar: die erste repräsentiert die kolline Form der Gesellschaft, während die beiden anderen der typischen, montanen Form angehören. Von diesen ist die Aufnahme 2. mit ihren azidophilen Trennarten als Dentario-Fagetum luzuletosum zu bezeichnen, während die Aufnahme 3. die typische Variante des Dentario-Fagetum typicum darstellt.

Der artenreiche Buchenwald ist im Untersuchungsgebiet als örtliche Dauergesellschaft aufzufassen. Bei der gesteinsbedingten Bodenarmut des Riesengebirges ist er nur auf besondere, nährstoffreiche Lagen beschränkt. Es sind meistens frische Mulden oder wasserzügige Rinnen, wo der Boden durch Berieselung vom fliessenden Wasser oder durch deluviale Anschlämmungen wesentlich eutrophiert wird. Viel seltener macht sich der Einfluss einer an sich reicheren Bodenunterlage bemerkbar. Günstige Nährstoff- und Wasserverhältnisse tragen zur Steigerung der biologischen Bodenaktivität bei. Die Humusform ist Mull; der Boden stellt meist eine eutrophe, reife Braunderde dar (s. Fig. 18.). 
Tabelle 12

Fragment eines Auenwaldes (Alno-Padion)

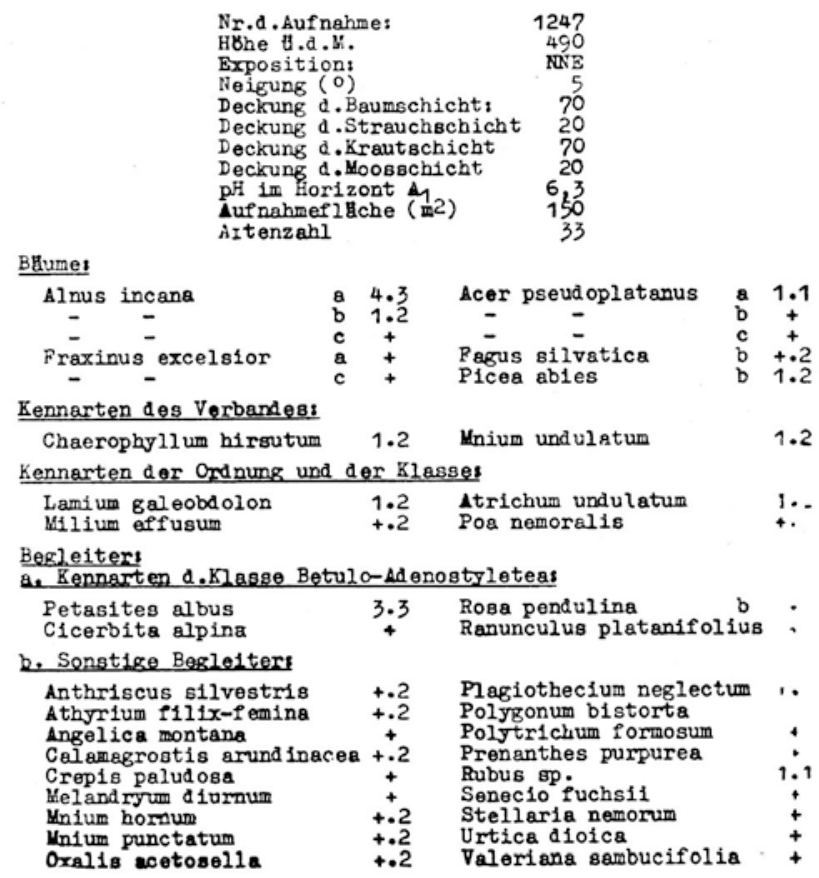

Verband: ALNO-PADION Kn a p p $1942 \mathrm{em}$. M a t. et Bor. 1957.

Im Gebiet des Nationalparks kommen nur ganz kleine Reste der auenwaldartigen Gesellschaften vor. Als Beispiel geben wir die Aufnahme eines relativ gut entwickelten Fragmenten an (s. Tab. 12.). Die Aufnahme stammt aus dem Kocheltal und stellt wahrscheinlich eine Durchdringung einer Alno-Padion-Gesellschaft mit der bachbegleitenden Hochstaudenflur (Petasitetum albi) dar. Bei der noch unzureichenden Kenntnis unserer Auenwälder, besonders in der submontan-montanen Stufie, lässt sich über die systematische Stellung solcher fragmentarischen Bestände kaum etwas sicheres sagen. 


\section{ZUSAMMENFASSUNG}

1. Als Folge einer pflanzensoziologischen Durchforschung der Waldgesellschaften des Riesengebirges sind 20 Vegetationseinheiten von 7 Assoziationen beschrieben worden.

2. Die bisher schon bekannten Vegetationstypen sind unter modernen Gesichtspunkten neugefasst und in ihrer systematischen Stellung geklärt worden.

3. Es wird gezeigt, dass die Fichtenbestände der unteren montanen Stufe, welche man bisjetzt als lauter künstliche Forste betrachtet hat, teilweise natürliche, lokalbedingte Dauergesellschaften darstellen und als eigene Assoziation aufzufassen sind, deren systematische Stellung allerdings noch weiterer Klärung bedarf.

4. Die ausgeschiedenen Einheiten wurden regional betrachtet und mit analogen Gesellschaften der Nachbargebiete verglichen.

5. Es wurde festgestellt, dass die Waldgesellschaften des Riesengebirges soziologisch-systematisch meist näher mit den Einheiten der mitteleuropäischen Mittelgebirge verwandt sind, als mit solchen der Westkarpathen. Nur die Knieholzgebüsche, welche sowohl in den Karpathen wie im Riesengebirge vorkommen, haben im Westen keine direkte Fortsetzung.

6. Einige untersuchten Gesellschaften kommen im Riesengebirge in besonderen regionalen Ausbildungen (Gebietsassoziationen oder geographischen Rassen) vor, was auf eine gewisse geobotanische Selbständigkeit der betreffenden Region hinweist.

7. Die Abhängigkeit der Vegetationsgliederung von dem Standort wurde in allen untersuchten Fällen festgestellt. Man kann für die ausgeschiedenen Einheiten ihre bestimmenden Standortsfaktoren angeben; ebenso lässt sich für jeden Standortstyp eine ihm entsprechende natürliche Gesellschaft nennen. In der Folge wird die Grundlage zur Feststellung der natürlichen potentiellen Vegetation des Gebietes geschaffen.

8. Die Vegetationsstufen wurden als Klimaxgebiete bestimmter Gesellschaften.aufgefasst. Als klimatisch bedingte Schlussgesellschaften des Riesengebirges sind in der unteren montanen Stufe das Luzulo-Fagetum montanum, in der oberen montanen Stufe das Piceetum hercynicum und in der subalpinen Knieholzstufe das Pinetum mughi sudeticum zu betrachten.

Botanisches Institut der Polnischen

Akademie der Wissenschaften

Abteilung für forstliche Vegetationskunde

Warszawa

(Eingegangen 13.4.1960) 


\section{LITERATUR}

B ra u n-B l anquet J., 1951, Pflanzensoziologie. Grundzüge der Vegetationskunde, 2. Aufl. - XI +631 pp. Springer-Verlag, Wien.

Braun-Blanquet J., S is singh G., V li e g e r J., 1939, Klasse der Vaccinio-Piceetea, Prodr. d. Pflanzengesellsch. 6: 1-123.

Doktorowicz-Hrebnicki S., 1948, Przeglądowa mapa geologiczna Polski. Wydanie A. Arkusz E1 Walbrzych, Państw. Inst. Geol., Warszawa.

Grüneberg H., S chlüt e r H., 1957, Waldgesellschaften im Thüringischen Schiefergebirge, Arch. f. Forstwesen. 6: 861-932. Berlin.

H u e c k K., 1939, Botanische Wanderungen im Riesengebirge, Pflanzensoziologie. 3. VIII + 116 pp. Verlag von G. Fischer, Jena.

K a we cki W., 1939, Lasy Żywiecczyzny, ich teraźniejszość i przeszlość (Zarys monograficzny), Prace Rolniczo-Leśne PAU, 35: 1-172, Kraków.

K u bi ën a W. L., 1953, Bestimmungsbuch und Systematik der Böden Europas, 392 pp. Ferdinand Enke Verlag, Stuttgart.

L a a t s c h W., 1954, Dynamik der mitteleuropäischen Mineralböden, XI + 277 pp., Verlag von Theodor Steinkopf, Dresden u. Leipzig.

L o h m e y e r W., T üx en R., 1958, Kurzer Bericht über die Exkursionen. - Bericht über das internationale Symposion Pflanzensoziologie, Bodenkunde vom 18. bis 22.9.1956 in Stolzenau/Wessr, Angew. Pflanzensoz. 15: 181-203, Stolzenau/Wessr.

M a c k o S., 1952, Zespoly roślinne w Karkonoszach. Cz. I. Karkonosze Wschodnie, Acta Sot. Bot. Polon. 21: 591-693, Warszawa.

M e dwecka-K ornaś A., 1955, Zespoly leśne Gorców, Ochrona Przyrody 23: $1-111$, Kraków.

M eusel H., 1954, Vegetationskundliche Studien über mitteleuropäische Waldgesellschaften. 4. Die Laubwaldgesellschaften des Harzgebietes. Angew. Pflanzensoz. Festschrift Aichinger. 1: 437-472, Wien.

M rá z K., 1957, Waldkundliche Untersuchungen im Mittelböhmischen Bergland und Erfahrungen mit der Anwendung statistischer Maschinen bei der synthetischen Bearbeitung, Archiv f. Forstwesen 6: 109-191, Berlin.

M y c zk ow ski S., 1958, Ochrona i przebudowa lasów Beskidu Malego, Ochrona Przyrody 25: 141-237, Kraków.

N oirfalise A., 1949, Premier aperçu sur l'étage du hêtre et les types de hêtraies en Haute-Ardenne, Bull. Inst. Agr. Stat. Rech. Gembloux., 17: 76-100.

Oberdorfer E., 1957, Süddeutsche Pflanzengesellschaften, Pflanzesoziologie 10. XXVIII + 564 pp. VEB Gustav Fischer Verlag, Jena.

Paw low ski B., S tecki K., 1927, Die Pflanzenassoziationen des Tatra-Gebirges. IV. Teil: Die Pflanzenassoziationen des Miętusia-Tales und des Hauptmassivs der Czerwone Wierchy, Bull. Acad. Polon. Sér. B. Suppl. 2. (1926): 79-121, Cracovie.

Paw lowski B., Sok olowski M., Walli s ch K., 1928, Die Pflanzenassoziationen des Tatra-Gebirges. VII. Teil. Die Pflanzenassoziationen und die Flora des Morskie Oko-Tales, Bull. Acad. Polon. Sér. B. Suppl. 2. (1927): 205--272, Cracovie.

Paw lowski B., Walas J., 1949, Les associations de plantes vasculaires des Monts de Czywczyn, Bull. Acad. Polon. Sér. B. (1948): 117-181, Cracovie.

Preis K., 1938, Ein Beitrag zur Kenntnis unserer Buchenwälder, Natur u. Heimat. 4: 106-111, Aussig. 
R e in hold F. 1939, Versuch einer Einteilung und Ubersicht der natürlichen Fichtenwälder (Piceion excelsae) Sachsens, Thar. Forstl. Jahrb. 90, 229, Berlin.

S c a m o n i A., Mitarb., 1958, Karte der natürlichen Vegetation, Klimaatlas d. Dtsch., Dem. Rep. 1. Ergänzungsband, Berlin.

Schlüter H., 1959, Waldgesellschaften und Wuchsbezirksgliederung im Grenzbe-reich der Eichen-Buchen- zur Buchenstufe am Nordwestabfall des Thüringer Waldes, Arch. f. Forstwesen, 8: 427-493, Berlin.

S e i be rt P., 1954, Die Wald- und Forstgesellschaften im Graf Görtzischen Forstbezirk Schlitz, Angew. Pflanzensoz., 9: 1-63, Stolzenau/Weser.

Sul m a T., 1929, Kosodrzewina i jej zespoly w Gorganach, Acta Soc. Bot. Polon. 6: 105-137, Warszawa.

Szafer W., Paw lowski B., Kulczyński S., 1923, Die Pflanzenassoziationen des Tatra-Gebirges. I. Teil: Die Pflanzenassoziationem des ChochołowskaTales, Bull. Acad. Polon. Sér. B. Suppl.: 1-65, Cracovie.

Szafer W., P a w low ski B., Kulczyń ski S., 1927, Die Pflanzenassoziationen des Tatra-Gebirges. III. Teil: Die Pflanzenassoziationen des Kościeliska-Tales, Bull. Acad. Polon. Sér. B. Suppl. 2. (1926): 13-78, Cracovie.

Trampler T., 1937, Kosodrzewina w Gorganach, Acta Soc. Bot. Polon. 14: 1-45, Warszawa.

Trautmann W., 1952, Pflanzensoziologische Untersuchungen der Fichtenwälder des Bayerischen Waldes, Forstwissenschaftl. Zentralblatt 71: 289-313.

Trautmann W., 1957, Natürliche Waldgesselschaften und nacheiszeitliche Waldgeschichte des Eggegebirges. Mitt. flor.-soz. Arbeitsgem. N. F. 6/7: 276-296, Stolzenau/Weser.

Tüx en R., 1937, Die Pflanzengesellschaften Nordwestdeutchlands, Mitt. flor.-soz. Arbeitsgem. Niedersachsen, 3: 1-170, Hannover.

$\mathrm{T}$ ü x e n R., 1954, Über die räumliche, durch Relief und Gestein bedingte Ordnung der natürlichen Waldgesellschaften am nördlichen Rande des Harzes. Vegetatio 5-6: $454-478$, Den Haag.

V olk O. H., Priehäus er G., 1938, Exkursion der Arbeitsgemeinschaft für forstliche Vegetationskunde in den Bayerischen Wald, Mscr.

W a l a s J., 1933, Roślinność Babiej Góry, Państwowa Rada Ochrony Przyrody, Monografie naukowe 2: $1-68$, Warszawa.

W i t ti g J., 1942/43, Die Laubwälder der Sudeten und ihres Vorlandes, Schlesiche Heimat, 1-21, Breslau, 1942/43.

Zlatnik A., 1925, Les associations de la végétation des Krkonoše et le pt. Věstn. Kral. Cesk. Společn. Nauk. 2: Praha.

Z lat nik A., 1928, Aperçu de la végétation des Krkonoše (Riesengebirge), Preslia 7: $94-152$, Praha. 